\title{
醋酸铜促进的酰胺-噁唑啉化合物与芳基硫醇的 $\mathrm{C}-\mathrm{H}$ 键双硫代化反应研究
}

\author{
王涛*, $a, b$ 王晓莎 $a$ 宋雅雯 $a$ 霍晶晶 $a$ 周敬栓 ${ }^{a}$ \\ 康庆伟 ${ }^{a} \quad$ 刘澜涛*, $a, b, c$ \\ ( ${ }^{a}$ 商丘师范学院化学化工学院 药物绿色合成河南省工程实验室 河南商丘 476000) \\ ${ }^{b}$ 商丘师范学院化学化工学院 河南省生物分子识别与传感重点实验室 河南商丘 476000) \\ ( ${ }^{c}$ 郑州大学化学学院 郑州 450001)
}

\begin{abstract}
摘要 以酰胺-噁唑啉为辅助基团, 在廉价的醋酸铜促进下, 实现了酰胺衍生物 $\mathrm{C}\left(\mathrm{sp}^{2}\right)$ - $\mathrm{H}$ 键与芳基硫醇 $\mathrm{S}$ 一 $\mathrm{H}$ 键的脱 氢偶联反应; 以中等到优秀的产率(最高可达 $90 \%$ )简单高效地合成了一系列双硫化的酰胺衍生物. 值得一提的是，底物 范围并不局限于各种取代苯基酰胺化合物，吡啶基酰胺化合物也可以兼容. 该反应的特点是：金属廉价、底物范围广、 反应条件温和、无需外加配体、空气作为氧化剂、区域选择性好(仅酰胺基团邻位的 $\mathrm{C}-\mathrm{H}$ 键发生反应，而噁唑啉基团 邻位的 $\mathrm{C}-\mathrm{H}$ 键不发生反应); 此外，克级规模的反应表明了其在合成中的实用性.
\end{abstract}

关键词 铜; $\mathrm{C}-\mathrm{H}$ 键; 硫代化; 酰胺-噁唑啉; 芳基硫醇

\section{$\mathrm{Cu}(\mathrm{OAc})_{2}$-Mediated $\mathrm{C}-\mathrm{H}$ Bond Dithiolation of Amide-Oxazolines with Aryl Thiols}

\author{
Wang, Tao ${ }^{*, a, b} \quad$ Wang, Xiaosha ${ }^{a} \quad$ Song, Yawen $^{a} \quad$ Huo, Jingjing $^{a} \quad$ Zhou, Jingshuan $^{a}$ \\ Kang, Qingwei ${ }^{a} \quad$ Liu, Lantao*,a,b,c \\ ( ${ }^{a}$ Henan Engineering Laboratory of Green Synthesis for Pharmaceuticals, School of Chemistry and \\ Chemical Engineering, Shangqiu Normal University, Shangqiu, Henan 476000) \\ $\left({ }^{b}\right.$ Henan Key Laboratory of Biomolecular Recognition and Sensing, School of Chemistry and \\ Chemical Engineering, Shangqiu Normal University, Shangqiu, Henan 476000) \\ ( ${ }^{c}$ College of Chemistry, Zhengzhou University, Zhengzhou 450001)
}

\begin{abstract}
An efficient copper-mediated dithiolation of $\mathrm{C}\left(\mathrm{sp}^{2}\right)-\mathrm{H}$ bonds with aryl thiols was achieved by using amide-oxazoline as directing group. This strategy gives a variety of functionalized thioethers in moderate to excellent yields (up to $90 \%$ ) in simple and efficient way. Importantly, the substrate scope is not limited to various substituted phenylamides, and diverse pyridine amides are also compatible. Furthermore, the protocol has been successfully implemented for the gram-scale synthesis as well.

Keywords copper; $\mathrm{C}-\mathrm{H}$ bond; thiolation; amide-oxazoline; aryl thiols
\end{abstract}

芳基硫醚骨架化合物广泛应用于生物活性化合 物 ${ }^{[1]}$ 、药物分子 ${ }^{[2]}$ (图 1)、有机合成 ${ }^{[3]}$ 、先进功能材料 ${ }^{[4]}$ 等相关领域. 因此, 开发高效简便构筑芳基硫醚骨架化
合物的新方法具有重要的学术意义和应用价值. 传统的 芳基硫醚骨架化合物的合成主要局限于有机卤化物或 有机金属化合物与統基亲核试剂的交叉偶联反应，该交

\footnotetext{
* Corresponding authors. E-mail: wt67751726@126.com; liult05@iccas.ac.cn

Received September 12, 2020; revised September 30, 2020; published online October 21, 2020.

Project supported by the Key Science Research of Education Committee in Henan Province (No. 19A150035), the Key Scientific and Technological Project of Henan Province (No. 192102110222), the Program for Science \& Technology Innovation Talents in Universities of Henan Province (No. 14HASTIT016) and the Program of Science and Technology Innovation Talents of Henan Province (No. 184100510011).

河南省高等学校重点科研(No. 19A150035)、河南省科技攻关(No. 192102110222)、河南省高校科技创新人才基金(No. 14HASTIT016)和河南省科技创 新杰出人才基金(No. 184100510011)资助项目.
} 
叉偶联反应的发生需要对底物进行预官能化、或额外使 用昂贵的配体、加入添加剂等, 并在反应过程中有大量 的副产物产生 ${ }^{[5]}$. 近年来, 过渡金属催化的 $\mathrm{C}-\mathrm{H}$ 活化 反应 ${ }^{[6]}$ 为芳基硫醚骨架化合物的合成提供了一种直接而 有效的途径. 各种过渡金属催化剂, 例如 $\mathrm{Pd}^{[7]} 、 \mathrm{Rh}^{[8]}$ 、 $\mathrm{Ru}^{[9]}$ 和 $\mathrm{Ni}^{[10]}$ 用于催化 $\mathrm{C}-\mathrm{H}$ 键硫代化反应已被报道.
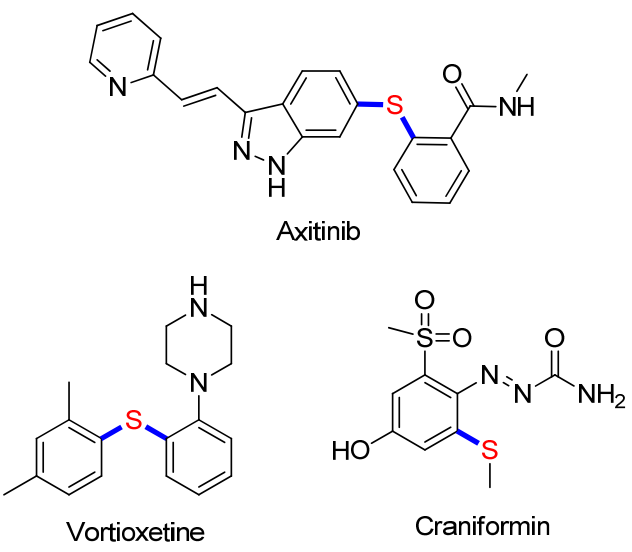

图 1 具有芳基硫醚骨架的药物分子

Figure 1 Drugs containing aryl thioethers skeleton

众所周知, 金属铜在自然界中含量丰富、价格低廉, 且其配合物种类繁多. 自从 2006 年 $Y u$ 和同事 ${ }^{[11]}$ 成功报 道铜催化的 2-苯吡啶与苯硫酚或二甲基二硫醚的 $\mathrm{C}-\mathrm{H}$ 键硫化的例子以来, 金属铜催化的直接 $\mathrm{C}-\mathrm{H}$ 键硫醚化 反应吸引了众多科学家的关注. Qing 课题组 ${ }^{[12]}$ 利用二甲 基亚砜(DMSO)作为甲基硫化试剂, 实现了铜催化的 2苯吡啶的 $\mathrm{C}-\mathrm{H}$ 键甲基硫化反应. 2012 年, Daugulis 等 ${ }^{[13]}$ 在铜催化的 $\mathrm{C}-\mathrm{H}$ 键硫醚化反应领域取得了重大突破, 即采用 8-氨基喹啉辅助双齿导向基团, 实现了 $\mathrm{C}-\mathrm{H}$ 键 三氟甲基磺酰化反应，反应条件温和，底物适用范围较 广, 并且 8-氨基喹啉导向基团反应之后很容易移除, 因 此为三氟甲基硫代酸衍生物的合成提供了一种高效的 合成策略. 最近, Song 课题组 ${ }^{[14]}$ 用 2-氨基烷基苯并咪唑 为双齿导向基与二硫化物实现了铜催化 $\mathrm{C}-\mathrm{H}$ 键硫代化 反应，该反应体系对于各种芳酰胺、烯基酰胺化合物均 能顺利进行. 另外, 以 2-(吡啶-2-基)异丙胺为导向基团, Shi 课题组 ${ }^{[15]}$ 发展了铜催化的 $\mathrm{C}-\mathrm{H}$ 键硫代化反应, 各 种硫代的杂芳烃衍生物被成功制备. 近些年, 尽管在铜 催化的 $\mathrm{C}-\mathrm{H}$ 键硫代化反应研究领域取得了重大进展, 但仍有一些问题值得挑战. 如与其他硫化试剂相比, 硫 醇化合物具有原料易得、成本低廉、便于操作等特点, 然 而硫醇化合物作为硫化试剂, 应用在直接 $\mathrm{C}-\mathrm{H}$ 键硫代 化反应或分子间 $\mathrm{C}-\mathrm{S}$ 键构建的文献报道较少 ${ }^{[16]}$. 另外, 从已知文献报道可以发现, 若使用大过量的铜盐作催化 剂, 单硫代化反应或双硫代化反应的反应区域选择性往
往很难调控.

最近我们课题组在碳一杂键的构筑方面取得了一些 研究结果 ${ }^{[17]}$. 为了进一步发展碳-杂键构筑新方法, 拓 展其应用范围，基于以上研究结果，本文考察了醋酸铜 促进的以酰胺-噁唑啉化合物为双齿导向基团与芳基硫 醇化合物进行 $\mathrm{C}-\mathrm{H}$ 键双硫代化反应(Scheme 1), 为 $\mathrm{C}-$ $\mathrm{S}$ 键的构筑及其芳基硫醚骨架化合物的合成开辟了新途 径.

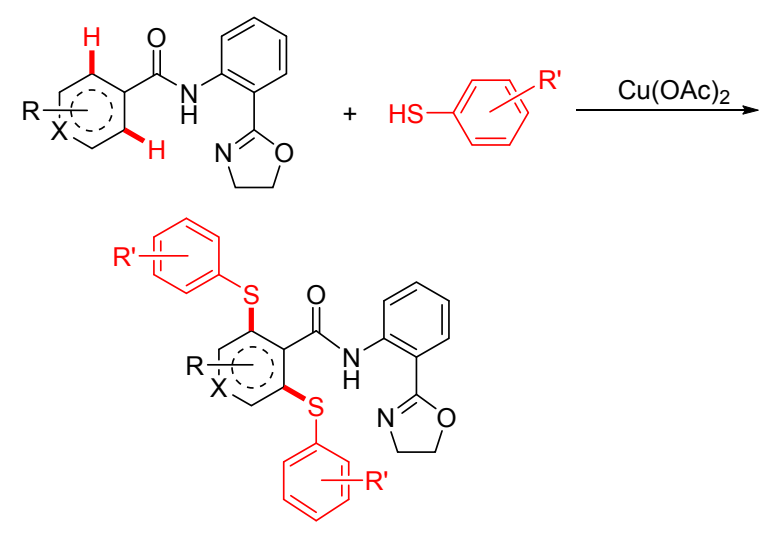

\footnotetext{
Abundant and low-cost copper catalyst

Dithiolation of amide-oxazoline

Broad substrates scope with various substituted phenylamides and pyridine amides

Gram-scale synthesis
}

图式 1 铜(II)促进的 C- $-\mathrm{H}$ 键双硫代化反应

Scheme $1 \mathrm{Cu}(\mathrm{II})$-mediated $\mathrm{C}-\mathrm{H}$ bond dhiolation.

\section{1 结果与讨论}

\section{1 反应条件考察}

首先选用酰胺-噁唑啉化合物 $1 \mathrm{a}(0.20 \mathrm{mmol})$ 与对 甲氧基苯硫酚(2a) (0.60 mmol)反应为模板反应，对反应 条件进行优化(表 1). 当使用 $\mathrm{Cu}(\mathrm{OAc})_{2}$ (1.0 equiv.), $\mathrm{NaOAc}(1.0$ equiv.)为碱, DMSO $(1.0 \mathrm{~mL})$ 为溶剂, 在 $100{ }^{\circ} \mathrm{C}$ 条件下反应 $15 \mathrm{~h}$, 可获得 $84 \%$ 分离收率的目标 产物 3aa(表 1, Entry 1), 化合物 3aa 的分子结构也经 X 单晶衍射进一步确认. 当将 $\mathrm{NaOAc}$ 换成 KOAc、CsOAc、 $\mathrm{K}_{2} \mathrm{CO}_{3}$ 等其他 7 种碱时, 发现反应产率大幅度下降(表 1 , Entries $2 \sim 8$ ), 说明碱的合理选取对该反应的顺利发生 起着至关重要的作用 ${ }^{[18]}$. 接着, 在 $\mathrm{NaOAc}$ (1.0 equiv.)为 碱的条件下对反应温度进行考察, 结果发现在 $80{ }^{\circ} \mathrm{C}$ 时 收率较好, 可达 90\%(表 1, Entry 10). 当使用 $\mathrm{Cu}(\mathrm{OAc})_{2}$ • $\mathrm{H}_{2} \mathrm{O}$ (1.0 equiv.)时，目标化合物的收率有所下降(表 1, Entry 12). 若换成 $\mathrm{Co}(\mathrm{OAc})_{2}$ 或 $\mathrm{Cu}(\mathrm{OAc})_{2} \bullet 4 \mathrm{H}_{2} \mathrm{O}$ 时, 没有 检测到相应的目标产物(表 1 , Entries 13 14). 最后将 $\mathrm{Cu}(\mathrm{OAc})_{2}$ 降低为 0.5 equiv. 或将反应时间缩短为 $12 \mathrm{~h}$, 结 果均不理想(表 1, Entries 15～16). 因此最终确定优化反 应条件为: 化合物 $\mathbf{1 a}(0.20 \mathrm{mmol})$, 化合物 $\mathbf{2 a}(0.60$ 
$\mathrm{mmol}$ ), $\mathrm{NaOAc}$ (1.0 equiv.), $\mathrm{Cu}(\mathrm{OAc})_{2}$ (1.0 equiv.), DMSO $(1.0 \mathrm{~mL})$, 反应 $15 \mathrm{~h}$.

表 1 反应条件篮选 ${ }^{a}$

Table 1 Optimization of the reaction conditions ${ }^{a}$

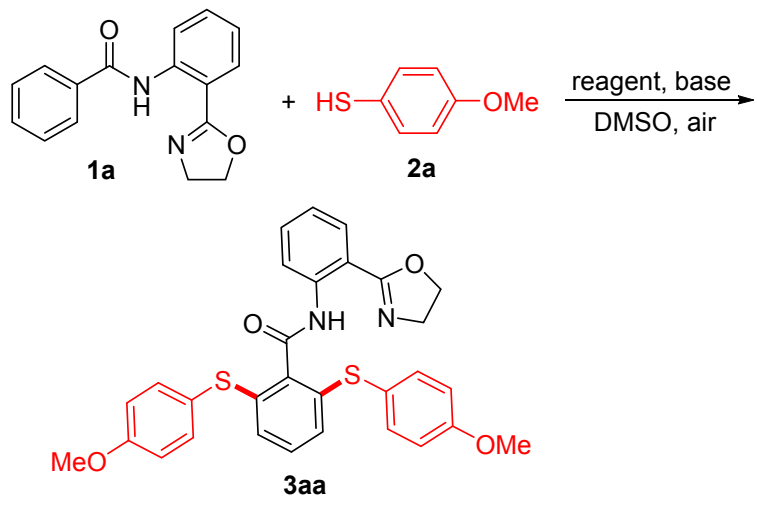

\begin{tabular}{clccc}
\hline Entry & \multicolumn{1}{c}{ Reagent } & Base & Temp. $/{ }^{\circ} \mathrm{C}$ & Yield $^{b} \%$ \\
\hline 1 & $\mathrm{Cu}(\mathrm{OAc})_{2}$ & $\mathrm{NaOAc}$ & 100 & 84 \\
2 & $\mathrm{Cu}(\mathrm{OAc})_{2}$ & $\mathrm{KOAc}$ & 100 & 20 \\
3 & $\mathrm{Cu}(\mathrm{OAc})_{2}$ & $\mathrm{CsOAc}$ & 100 & 30 \\
4 & $\mathrm{Cu}(\mathrm{OAc})_{2}$ & $\mathrm{~K}_{2} \mathrm{CO}_{3}$ & 100 & 24 \\
5 & $\mathrm{Cu}(\mathrm{OAc})_{2}$ & $\mathrm{Na}_{2} \mathrm{CO}_{3}$ & 100 & 8 \\
6 & $\mathrm{Cu}(\mathrm{OAc})_{2}$ & $\mathrm{Cs}_{2} \mathrm{CO}_{3}$ & 100 & Trace \\
7 & $\mathrm{Cu}(\mathrm{OAc})_{2}$ & $\mathrm{NaHCO}_{3}$ & 100 & 26 \\
8 & $\mathrm{Cu}(\mathrm{OAc})_{2}$ & $\mathrm{~K}_{3} \mathrm{PO}_{4}$ & 100 & 15 \\
9 & $\mathrm{Cu}(\mathrm{OAc})_{2}$ & $\mathrm{NaOAc}_{2}$ & 120 & 76 \\
10 & $\mathrm{Cu}(\mathrm{OAc})_{2}$ & $\mathrm{NaOAc}_{2}$ & 80 & 90 \\
11 & $\mathrm{Cu}(\mathrm{OAc})_{2}$ & $\mathrm{NaOAc}$ & 60 & 59 \\
12 & $\mathrm{Cu}(\mathrm{OAc})_{2} \bullet \mathrm{H}_{2} \mathrm{O}$ & $\mathrm{NaOAc}$ & 80 & 82 \\
13 & $\mathrm{Co}(\mathrm{OAc})_{2}$ & $\mathrm{NaOAc}$ & 80 & - \\
14 & $\mathrm{Cu}(\mathrm{OAc})_{2} \bullet 4 \mathrm{H}_{2} \mathrm{O}$ & $\mathrm{NaOAc}$ & 80 & - \\
$15^{c}$ & $\mathrm{Cu}(\mathrm{OAc})_{2}$ & $\mathrm{NaOAc}$ & 80 & 30 \\
$16^{d}$ & $\mathrm{Cu}(\mathrm{OAc})_{2}$ & $\mathrm{NaOAc}$ & 80 & 73 \\
\hline $\mathrm{All}$ &
\end{tabular}

${ }^{a}$ All reactions were carried out using 1a $(0.20 \mathrm{mmol}), \mathbf{2 a}(0.60 \mathrm{mmol})$, base (1.0 equiv.), plus reagent(1.0 equiv.) in DMSO $(1.0 \mathrm{~mL})$ for $15 \mathrm{~h} .{ }^{b}$ Isolated yields. ${ }^{c} \mathrm{Cu}(\mathrm{OAc})_{2}\left(0.5\right.$ equiv.). ${ }^{d}$ Reaction time was $12 \mathrm{~h}$.

\section{2 各种取代苯基酰胺-噁唑啉化合物底物拓展}

在上述优化的反应条件下，首先考察了各种取代苯 基酰胺-噁唑啉化合物与对甲氧基苯硫酚的 $\mathrm{C}-\mathrm{H}$ 键双 硫代化反应(表 2). 结果发现酰胺-噁唑啉化合物苯环上 带有不同电性的取代基对该反应均具有兼容性(产率 51\% 90\%). 但是相比而言, 供电子取代的苯基酰胺噁唑啉化合物与对甲氧基苯硫酚的 $\mathrm{C}-\mathrm{H}$ 键双硫代化反 应活性更高 (3ab 3af $v s$ 3ag 3ah, 3ai $\sim 3 a j$ j $v s$ 3ak). 间 位取代的苯基酰胺一腎唑啉化合物与对甲氧基苯硫酚进 行 $\mathrm{C}-\mathrm{H}$ 键双硫代化反应时, 反应活性没有明显变化, 目标产物可以获得 66\% 84\%的收率(3ai 3ak).

\section{3 芳基硫醇底物拓展}

醋酸铜用于各种取代苯基酰胺-噁唑啉化合物与对 甲氧基苯硫酚的 $\mathrm{C}-\mathrm{H}$ 键双硫代化反应, 表现出较好的
催化活性和底物普适性. 在上述实验的基础上继续探究 苯甲酰胺-噁唑啉化合物与芳基硫酚的 $\mathrm{C}-\mathrm{H}$ 键双硫代 化反应, 实验结果如表 3 所示. 不同取代基的芳基硫酚 发生 $\mathrm{C}-\mathrm{H}$ 键双硫代化反应. 均能以中等至较高的收率 得到芳基硫梄骨架衍生物(48\% 80\%, 3ba 3bg). 相比 而言, 苯环上带有供电子基团的芳基硫酚发生 $\mathrm{C}-\mathrm{H}$ 键 双硫代化反应的活性优于苯环上带有吸电子基团的芳 基硫酚(3ba 3bc vs 3bd). 另外，邻甲氧基苯硫酚发生 $\mathrm{C}-\mathrm{H}$ 键双硫代化反应时，目标产物的产率有所下降 $(70 \%, 3 b f)$, 可能的原因为空间位阻对该反应产生了影 响.

\subsection{4-吡啶基酰胺-噁唑啉化合物与芳基硫醇的 $\mathrm{C}-\mathrm{H}$ 键双硫代化反应底物拓展}

根据文献报道得知 ${ }^{[15]}$, 吡啶基酰胺类化合物发生 $\mathrm{C}-\mathrm{H}$ 键硫代化反应通常存在底物范围窄，催化效率较 低等问题. 其可能的原因至少有以下两个方面: (1)硫醇 化合物易于氧化，在氧化过程中通常容易和金属催化剂 进行配位，致使金属催化剂失活; (2)杂芳基上的杂原子 与金属催化剂产生强配位，可能会与导向基团杂原子竞 争, 阻止所需要的 $\mathrm{C}-\mathrm{H}$ 键发生活化反应. 基于上述实 验结果, 把研究的焦点转移到了杂芳基酰胺-噁唑啉化 合物与芳基硫酚的 $\mathrm{C}-\mathrm{H}$ 键双硫代化反应(表 4). 令人可 喜的是，当使用吡啶基酰胺一噁唑啉化合物与芳基硫酚 发生 $\mathrm{C}-\mathrm{H}$ 键双硫代化反应时，均能以中等的收率得到 相应的目标产物(37\% 61\%, 5a $\sim 5 \mathbf{f})$. 相比而言，吡啶 基酰胺一噁唑啉化合物与芳基硫酚发生 $\mathrm{C}-\mathrm{H}$ 键双硫代 化反应时反应活性略微下降. 总体而言，该反应为一系 列功能化的硫醚化合物的制备提供了一种简便有效的 新方法.

\section{5 克级规模合成应用}

为了进一步探索该方法在工业化生产中的应用潜 力, 进行了克级规模合成实验(Scheme 2). 在本文优化
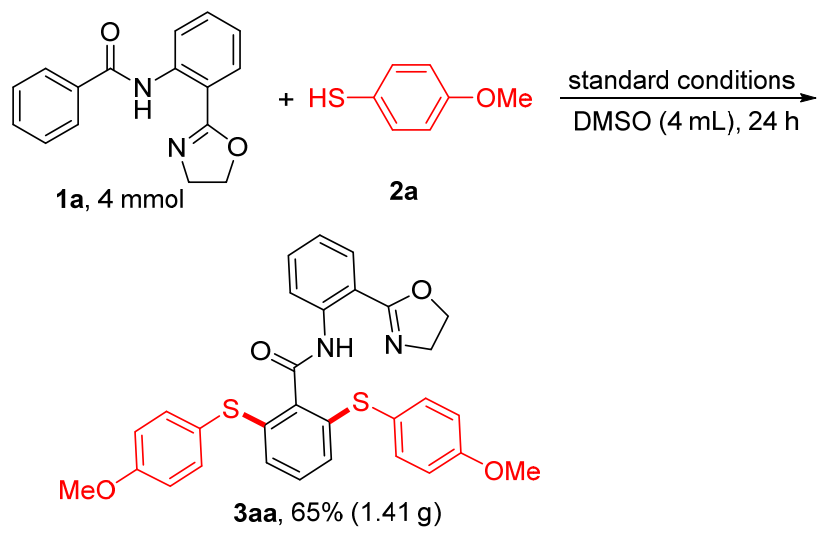

图式 2 克级规模合成

Scheme 2 Gram-scale synthesis 
表 2 各种取代苯基酰胺类化合物底物拓展 ${ }^{a}$

Table 2 Scope of the reaction in terms of various substituted phenylamides ${ }^{a}$
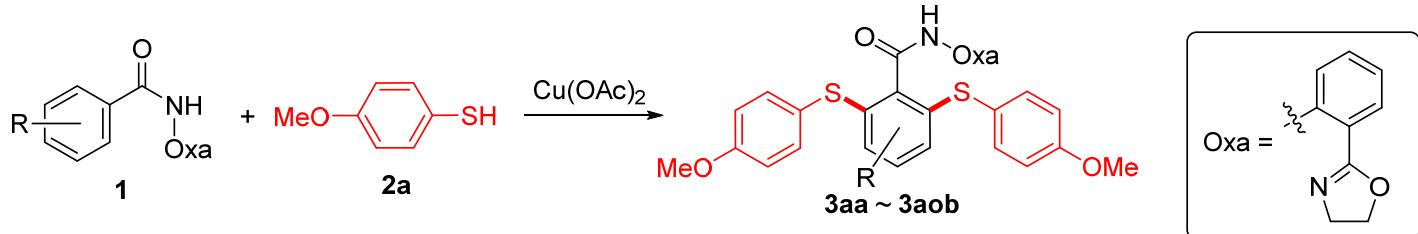

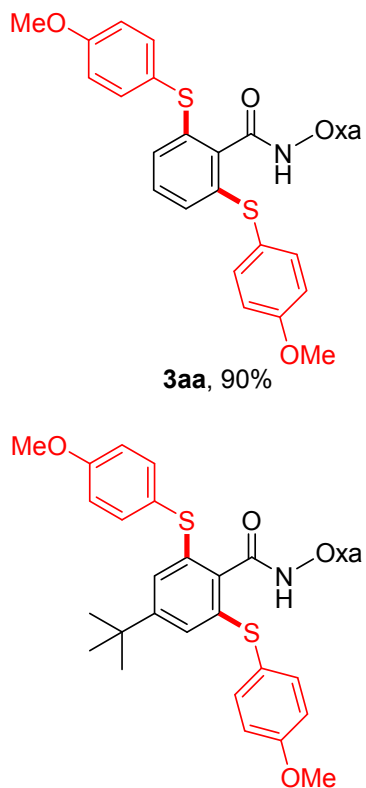

3ad, $73 \%$<smiles>COc1ccc(Sc2cc(Br)cc(Sc3ccc(OC)cc3)c2C(=O)NOC(C)C)cc1</smiles>

$3 \mathrm{ah}, 55 \%$

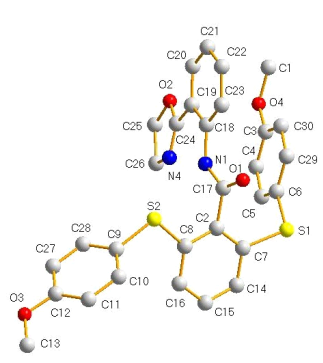

$\mathrm{MeO}$

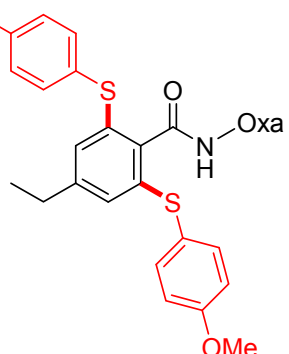

3ae, $84 \%$

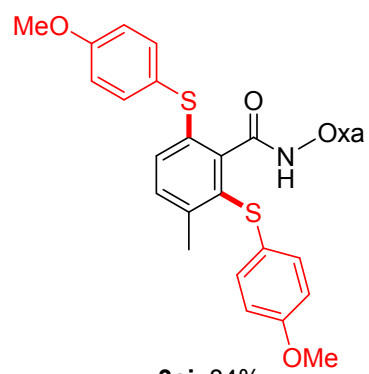

$3 a i, 84 \%$

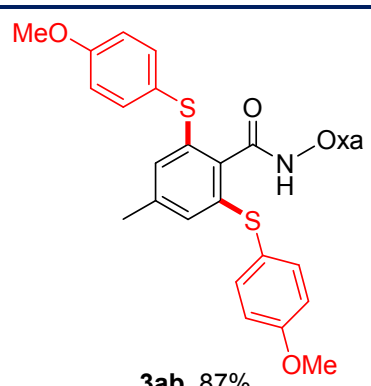

$3 a b, 87 \%$

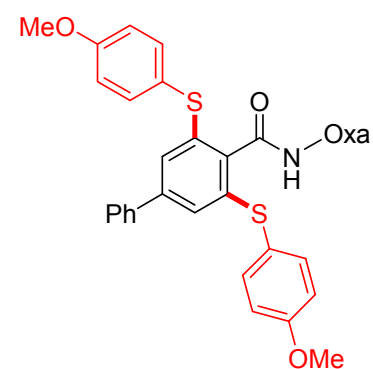

3af, $83 \%$

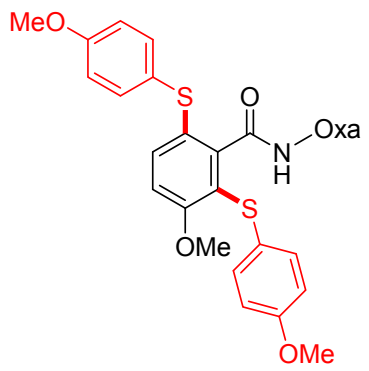

3aj, 72\%

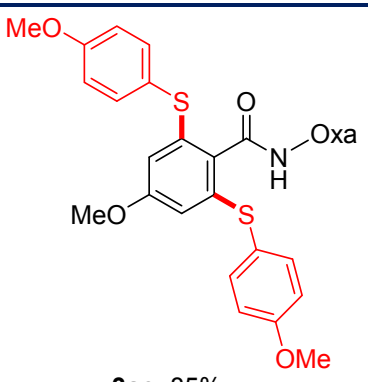

3ac, $85 \%$

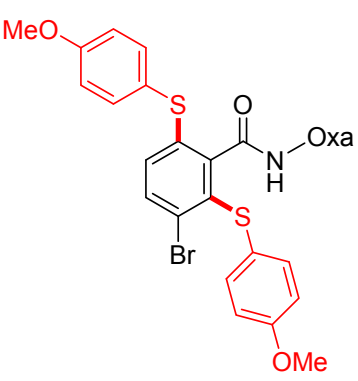

3al, $61 \%$

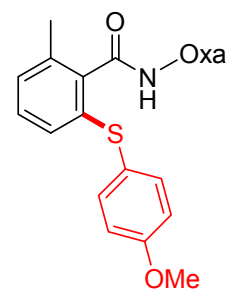

3am, 68\%

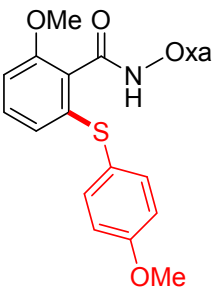

3an, $43 \%$

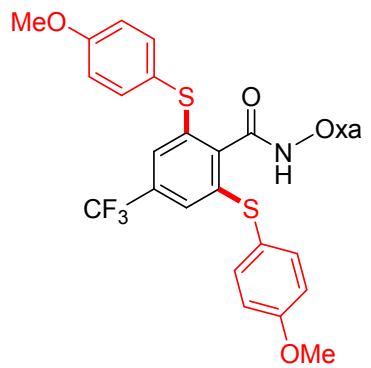

3ag, $51 \%$

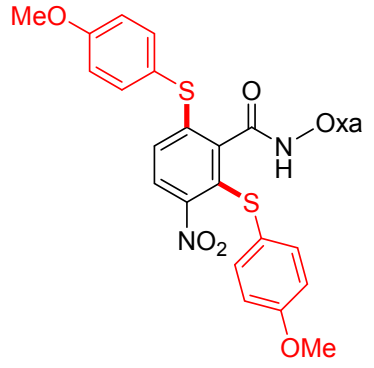

3ak, 66\%

${ }^{a}$ All reactions were carried out using $1(0.20 \mathrm{mmol}), 2 \mathrm{a}(0.60 \mathrm{mmol}), \mathrm{NaOAc}(1.0$ equiv. $)$, plus $\left.\mathrm{Cu}(\mathrm{OAc})\right)_{2}(1.0$ equiv. $)$ in DMSO (1.0 mL) at $80{ }^{\circ} \mathrm{C}$ for $15 \mathrm{~h} .{ }^{b} \mathrm{Iso}-$ lated yields.

的条件下将反应溶剂的用量改为 $4 \mathrm{~mL}$, 反应时间延长 至 $24 \mathrm{~h}$ ，苯甲酰胺-噁唑啉化合物 $\mathbf{1 a}$ 与对甲氧基苯硫酚 (2a)的 $\mathrm{C}-\mathrm{H}$ 键双硫代化反应, 仍然可以得到比较理想 的结果(Scheme 2, 3aa, 65\%, $1.41 \mathrm{~g}$ ), 这一结果进一步证
实了该方法在有机合成中的应用潜力.

\section{2 结论}

在醋酸铜促进下，实现了酰胺-噁唑啉化合物与芳 
表 3 芳基硫醇类化合物底物拓展 ${ }^{a}$

Table 3 Scope of the reaction in terms of aryl thiols ${ }^{a}$

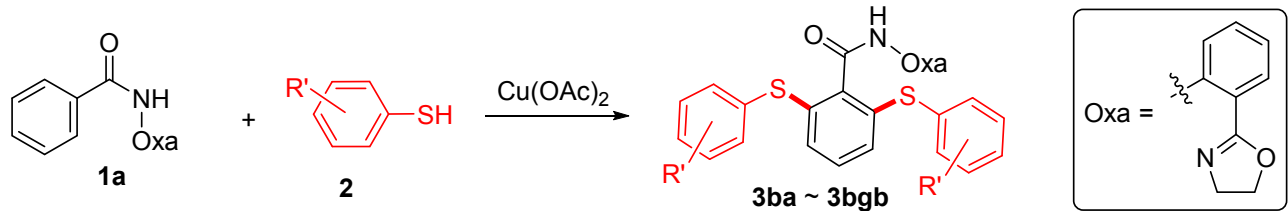

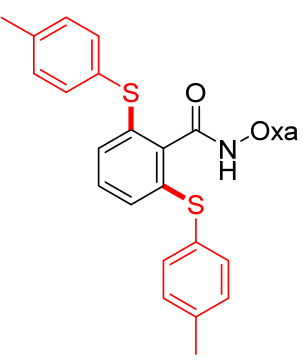

3ba, $70 \%$

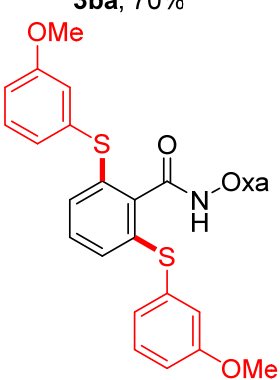

3be, $80 \%$

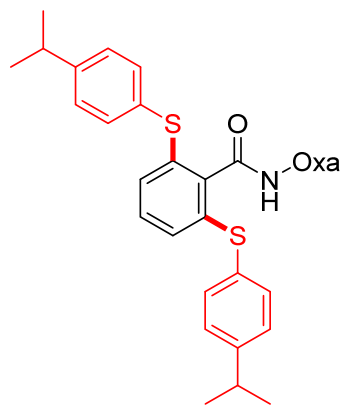

3bb, $61 \%$

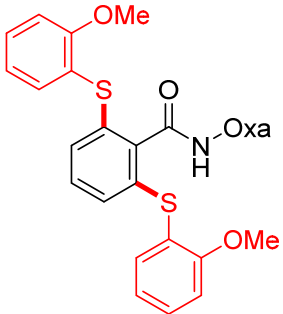

3bf, $70 \%$

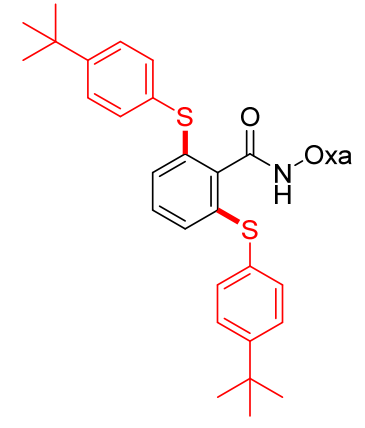

$3 b c, 63 \%$

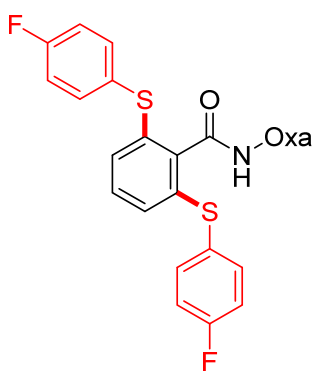

3bd, $48 \%$

${ }^{a}$ All reactions were carried out using 1a $(0.20 \mathrm{mmol}), 2(0.60 \mathrm{mmol}), \mathrm{NaOAc}(1.0$ equiv. $)$, plus $\mathrm{Cu}(\mathrm{OAc})_{2}(1.0$ equiv. $)$ in DMSO $(1.0 \mathrm{~mL})$ at $80{ }^{\circ} \mathrm{C}$ for $15 \mathrm{~h} .{ }^{b} \mathrm{Iso}-$ lated yields.

表 4 吡啶基酰胺类化合物底物拓展

Table 4 Scope of the reaction in terms of diverse pyridine amides ${ }^{a}$
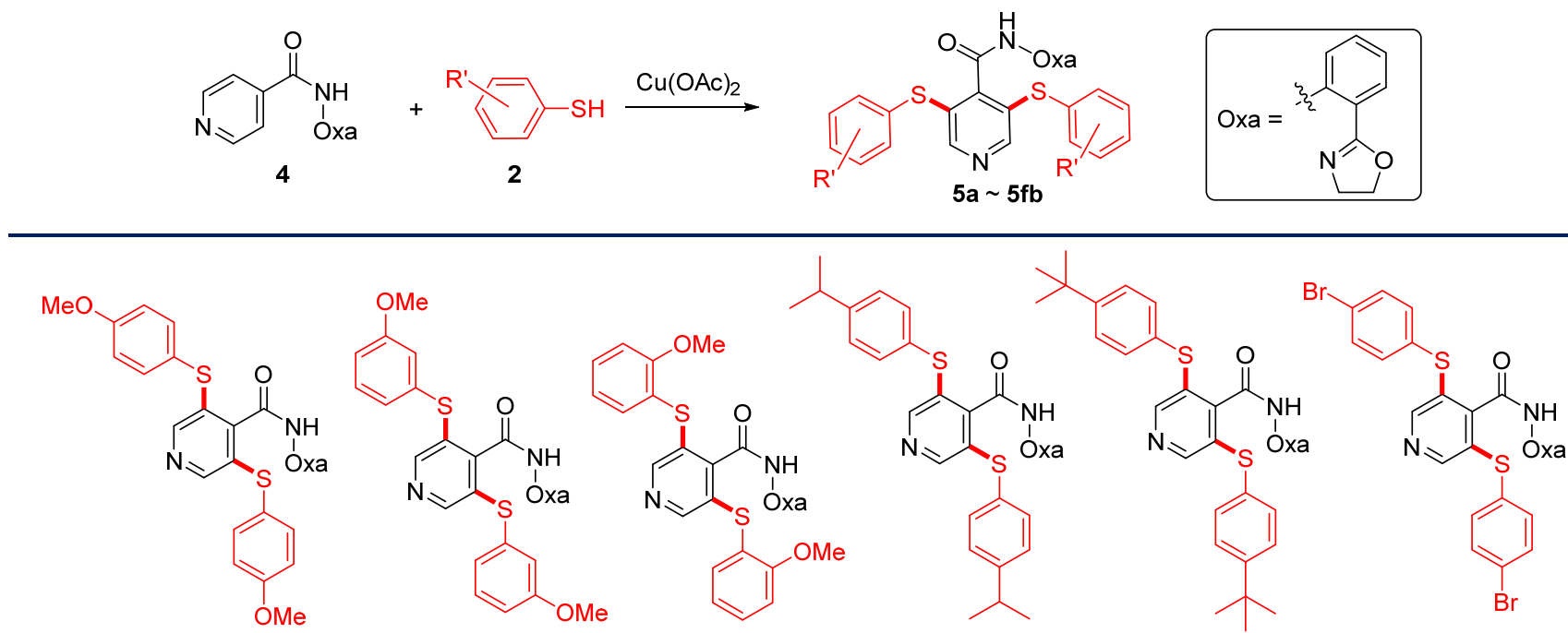

5a, $48 \%$

$5 b, 47 \%$

5c, $37 \%$

5d, $44 \%$

5e, $46 \%$

5f, $61 \%$

${ }^{a}$ All reactions were carried out using $4(0.20 \mathrm{mmol}), 2(0.60 \mathrm{mmol}), \mathrm{NaOAc}(1.0$ equiv. $)$, plus $\mathrm{Cu}(\mathrm{OAc})_{2}(1.0$ equiv. $)$ in DMSO $(1.0 \mathrm{~mL})$ at $80{ }^{\circ} \mathrm{C}$ for $15 \mathrm{~h} .{ }^{b}$ Isolated yields.

基硫醇化合物的 $\mathrm{C}-\mathrm{H}$ 键双硫代化反应. 该方法具有反 应条件简单、底物适用范围较广、区域选择性较好等特
点. 在优化的反应条件下, 各种取代苯基酰胺-噁唑啉 化合物、吡啶基酰胺-噁唑啉化合物均能与芳基硫醇发 
生 $\mathrm{C}-\mathrm{H}$ 键硫代反应, 得到相应的双硫代芳基硫醚骨架 化合物. 另外, 芳基酰胺一噁唑啉化合物及其他双齿导 向基化合物的设计合成与 $\mathrm{C}-\mathrm{H}$ 键活化反应目前正在研 究中.

\section{3 实验部分}

\section{1 仪器与试剂}

${ }^{1} \mathrm{H}$ NMR (400 MHz) 和 ${ }^{13} \mathrm{C} \mathrm{NMR}(100 \mathrm{MHz}$ )采用 Bruker AV 400 核磁共振仪测定, 所用溶剂为 $\mathrm{CDCl}_{3}$, TMS 为内标. 柱层析使用 $200 \sim 300$ 目硅胶, 实验所用 试剂均为市售分析纯试剂. 酰胺-噁唑啉化合物按照前 期文献报道的方法合成 ${ }^{[19]}$.

\section{2 实验方法}

空气氛围下, $15 \mathrm{~mL}$ Schlenk 反应管加入 $0.20 \mathrm{mmol}$ 酰胺-噁唑啉化合物, $0.60 \mathrm{mmol}$ 芳基硫醇, $0.20 \mathrm{mmol}$ 醋 酸铜, $0.20 \mathrm{mmol}$ 醋酸钠, 再加入 $1.0 \mathrm{~mL}$ DMSO 作为溶 剂, $80{ }^{\circ} \mathrm{C}$ 条件下反应 $15 \mathrm{~h}$. 反应结束后, 停止加热、萃 取、旋蒸浓缩，柱层析分离得到相应的目标产物.

$N$-(2-(4,5-二氢噁唑-2-基)苯基)-2,6-双((4-甲氧基苯 基)硫代)苯甲酰胺(3aa): 浅黄色油状液体. $97.7 \mathrm{mg}$, 产 率 90\%. ${ }^{1} \mathrm{H}$ NMR $\left(400 \mathrm{MHz}, \mathrm{CDCl}_{3}\right) \delta: 12.48$ (s, 1H), 9.01 $(\mathrm{d}, J=8.4 \mathrm{~Hz}, 1 \mathrm{H}), 7.87$ (d, $J=7.8 \mathrm{~Hz}, 1 \mathrm{H}), 7.53$ (t, $J=7.8$ $\mathrm{Hz}, 1 \mathrm{H}), 7.38$ (d, $J=8.6 \mathrm{~Hz}, 4 \mathrm{H}), 7.12$ (t, $J=7.6 \mathrm{~Hz}, 1 \mathrm{H})$, 7.06 (t, $J=7.9 \mathrm{~Hz}, 1 \mathrm{H}), 6.94$ (d, $J=7.8 \mathrm{~Hz}, 2 \mathrm{H}), 6.80$ (d, $J=8.5 \mathrm{~Hz}, 4 \mathrm{H}), 4.32(\mathrm{t}, J=9.4 \mathrm{~Hz}, 2 \mathrm{H}), 3.98(\mathrm{t}, J=9.5$ $\mathrm{Hz}, 2 \mathrm{H}), 3.76(\mathrm{~s}, 6 \mathrm{H}) ;{ }^{13} \mathrm{C} \mathrm{NMR}\left(100 \mathrm{MHz}, \mathrm{CDCl}_{3}\right) \delta$ : $165.9,164.3,159.8,139.6,138.7,136.9,135.5,132.6$, $129.6,129.1,128.4,124.6,122.7,120.3,114.8,113.7$, 66.1, 55.3, 54.8. HRMS (ESI) calcd for $\mathrm{C}_{30} \mathrm{H}_{27} \mathrm{~N}_{2} \mathrm{O}_{4} \mathrm{~S}_{2}$ $[\mathrm{M}+\mathrm{H}]^{+}$543.1407, found 543.1406.

$N$-(2-(4,5-二氢噁唑-2-基)苯基)-2,6-双((4-甲氧基苯 基)硫代)-4-甲基苯甲酰胺(3ab): 浅黄色油状液体. 96.9 $\mathrm{mg}$, 产率 87\%. ${ }^{1} \mathrm{H}$ NMR (400 $\mathrm{MHz}, \mathrm{CDCl}_{3}$ ) $\delta: 12.40$ (s, $1 \mathrm{H}), 8.99$ (d, $J=8.4 \mathrm{~Hz}, 1 \mathrm{H}), 7.85$ (d, $J=7.6 \mathrm{~Hz}, 1 \mathrm{H}), 7.51$ (t, $J=7.6 \mathrm{~Hz}, 1 \mathrm{H}), 7.37(\mathrm{~d}, J=8.5 \mathrm{~Hz}, 4 \mathrm{H}), 7.10(\mathrm{t}, J=7.5$ $\mathrm{Hz}, 1 \mathrm{H}), 6.79 \sim 6.77(\mathrm{~m}, 6 \mathrm{H}), 4.30(\mathrm{t}, J=9.5 \mathrm{~Hz}, 2 \mathrm{H}), 3.96$ $(\mathrm{t}, J=9.5 \mathrm{~Hz}, 2 \mathrm{H}), 3.75(\mathrm{~s}, 6 \mathrm{H}), 2.12(\mathrm{~s}, 3 \mathrm{H}) ;{ }^{13} \mathrm{C} \mathrm{NMR}$ $\left(100 \mathrm{MHz}, \mathrm{CDCl}_{3}\right) \delta: 166.2,164.2,159.7,139.8,139.6$, $136.8,136.2$, 135.2, 132.6, 129.6, 129.1, 124.9, 122.6, 120.2, 114.8, 113.6, 66.1, 55.3, 54.9, 53.5, 21.3. HRMS (ESI) calcd for $\mathrm{C}_{31} \mathrm{H}_{29} \mathrm{~N}_{2} \mathrm{O}_{4} \mathrm{~S}_{2}[\mathrm{M}+\mathrm{H}]^{+}$557.1563, found 557.1563.

$N$-(2-(4,5-二氢噁唑-2-基)苯基)-4-甲氧基-2,6-双((4甲氧基苯基)硫代)苯甲酰胺(3ac): 浅黄色油状液体. 97.4 $\mathrm{mg}$, 产率 85\%. ${ }^{1} \mathrm{H} \mathrm{NMR}\left(400 \mathrm{MHz}, \mathrm{CDCl}_{3}\right) \delta: 12.46$ (s,
1H), $9.00(\mathrm{~d}, J=8.2 \mathrm{~Hz}, 1 \mathrm{H}), 7.85$ (d, $J=7.6 \mathrm{~Hz}, 1 \mathrm{H}), 7.51$ (t, $J=7.5 \mathrm{~Hz}, 1 \mathrm{H}), 7.39$ (d, $J=8.1 \mathrm{~Hz}, 4 \mathrm{H}), 7.10$ (t, $J=7.4$ $\mathrm{Hz}, 1 \mathrm{H}), 6.80$ (d, $J=8.1 \mathrm{~Hz}, 4 \mathrm{H}), 6.40(\mathrm{~s}, 2 \mathrm{H}), 4.31(\mathrm{t}, J=$ $9.3 \mathrm{~Hz}, 2 \mathrm{H}), 4.00(\mathrm{t}, J=9.3 \mathrm{~Hz}, 2 \mathrm{H}), 3.75(\mathrm{~s}, 6 \mathrm{H}), 3.53$ (s, $3 \mathrm{H}) ;{ }^{13} \mathrm{C}$ NMR $\left(100 \mathrm{MHz}, \mathrm{CDCl}_{3}\right) \delta: 165.9,164.3,160.0$, $159.8,139.7,138.7,135.8,132.6,131.0,129.1,124.0$, 122.6, 120.2, 114.9, 113.6, 113.2, 66.1, 55.3, 55.2, 54.9. HRMS (ESI) calcd for $\mathrm{C}_{31} \mathrm{H}_{29} \mathrm{~N}_{2} \mathrm{O}_{5} \mathrm{~S}_{2}[\mathrm{M}+\mathrm{H}]^{+}$573.1512, found 573.1513 .

4-(叔丁基)- $N$-(2-(4,5-二氢噁唑-2-基)苯基)-2,6-双 ((4-甲氧基苯基)硫代)苯甲酰胺(3ad): 浅黄色油状液体. $87.4 \mathrm{mg}$, 产率 73\%. ${ }^{1} \mathrm{H} \mathrm{NMR}\left(400 \mathrm{MHz}, \mathrm{CDCl}_{3}\right) \delta: 12.36$ (s, 1H), 8.99 (d, $J=8.3 \mathrm{~Hz}, 1 \mathrm{H}), 7.83$ (dd, $J=7.9 \mathrm{~Hz}, 1.4$ $\mathrm{Hz}, 1 \mathrm{H}), 7.52 \sim 7.48(\mathrm{~m}, 1 \mathrm{H}), 7.37 \sim 7.33(\mathrm{~m}, 4 \mathrm{H}), 7.12 \sim$ $7.08(\mathrm{~m}, 1 \mathrm{H}), 7.05(\mathrm{~s}, 2 \mathrm{H}), 6.79 \sim 6.75(\mathrm{~m}, 4 \mathrm{H}), 4.28(\mathrm{t}$, $J=9.5 \mathrm{~Hz}, 2 \mathrm{H}), 3.94(\mathrm{t}, \quad J=9.4 \mathrm{~Hz}, 2 \mathrm{H}), 3.74(\mathrm{~s}, 6 \mathrm{H})$, $1.10(\mathrm{~s}, 9 \mathrm{H}) ;{ }^{13} \mathrm{C}$ NMR $\left(100 \mathrm{MHz}, \mathrm{CDCl}_{3}\right) \delta: 166.2,164.1$, $159.5,152.7,139.6,136.9,135.8,134.9,132.5,129.0$, 126.7, 125.2, 122.6, 120.3, 114.7, 113.7, 66.1, 55.3, 54.9, 34.9, 30.9. HRMS (ESI) calcd for $\mathrm{C}_{34} \mathrm{H}_{35} \mathrm{~N}_{2} \mathrm{O}_{4} \mathrm{~S}_{2}[\mathrm{M}+\mathrm{H}]^{+}$ 599.2033 , found 599.2033.

$N$-(2-(4,5-二氢噁唑-2-基)苯基)-4-乙基-2,6-双((4-甲 氧基苯基)硫代)苯甲酰胺(3ae): 浅黄色油状液体. 95.9 $\mathrm{mg}$, 产率 84\%. ${ }^{1} \mathrm{H} \mathrm{NMR}\left(400 \mathrm{MHz}, \mathrm{CDCl}_{3}\right) \delta: 12.39$ (s, $1 \mathrm{H}), 8.99$ (d, $J=8.3 \mathrm{~Hz}, 1 \mathrm{H}), 7.84$ (dd, $J=7.9 \mathrm{~Hz}, 1.3 \mathrm{~Hz}$, $1 \mathrm{H}), 7.52 \sim 7.48(\mathrm{~m}, 1 \mathrm{H}), 7.36(\mathrm{~d}, J=8.8 \mathrm{~Hz}, 4 \mathrm{H}), 7.12 \sim$ $7.08(\mathrm{~m}, 1 \mathrm{H}), 6.84(\mathrm{~s}, 2 \mathrm{H}), 6.77(\mathrm{~d}, J=8.8 \mathrm{~Hz}, 4 \mathrm{H}), 4.29$ (t, $J=9.5 \mathrm{~Hz}, 2 \mathrm{H}), 3.95(\mathrm{t}, J=9.6 \mathrm{~Hz}, 2 \mathrm{H}), 3.75(\mathrm{~s}, 6 \mathrm{H}), 2.42$ (q, $J=7.6 \mathrm{~Hz}, 2 \mathrm{H}), 1.04(\mathrm{t}, J=7.6 \mathrm{~Hz}, 3 \mathrm{H}) ;{ }^{13} \mathrm{C}$ NMR $(100$ $\left.\mathrm{MHz}, \mathrm{CDCl}_{3}\right) \delta: 166.2,164.2,159.6,145.9,139.6,137.1$, $136.2,135.1,132.5,129.1,128.7,125.0,122.6,120.2$, 114.7, 113.6, 66.1, 55.3, 54.9, 28.5, 15.1. HRMS (ESI) calcd for $\mathrm{C}_{32} \mathrm{H}_{31} \mathrm{~N}_{2} \mathrm{O}_{4} \mathrm{~S}_{2}[\mathrm{M}+\mathrm{H}]{ }^{+}$571.1720, found 571.1721 .

$N$-(2-(4,5-二氢噁唑-2-基)苯基)-3,5-双((4-甲氧基苯 基)硫代)-[1,1'-联苯]-4-甲酰胺(3af): 浅黄色油状液体. $102.7 \mathrm{mg}$, 产率 $83 \%$. ${ }^{1} \mathrm{H} \mathrm{NMR}\left(400 \mathrm{MHz}, \mathrm{CDCl}_{3}\right) \delta$ : 12.52 (s, 1H), 9.02 (d, $J=8.4 \mathrm{~Hz}, 1 \mathrm{H}), 7.86$ (dd, $J=7.9$ $\mathrm{Hz}, 1.4 \mathrm{~Hz}, 1 \mathrm{H}), 7.54 \sim 7.50(\mathrm{~m}, 1 \mathrm{H}), 7.41(\mathrm{~d}, J=8.8 \mathrm{~Hz}$, $4 \mathrm{H}), 7.33 \sim 7.28(\mathrm{~m}, 5 \mathrm{H}), 7.20(\mathrm{~s}, 2 \mathrm{H}), 7.13 \sim 7.11(\mathrm{~m}$, $1 \mathrm{H}), 6.78$ (d, $J=8.8 \mathrm{~Hz}, 4 \mathrm{H}), 4.30(\mathrm{t}, J=9.5 \mathrm{~Hz}, 2 \mathrm{H}), 3.97$ $(\mathrm{t}, J=9.5 \mathrm{~Hz}, 2 \mathrm{H}), 3.73(\mathrm{~s}, 6 \mathrm{H}) ;{ }^{13} \mathrm{C} \mathrm{NMR}(100 \mathrm{MHz}$, $\left.\mathrm{CDCl}_{3}\right) \delta: 165.9,164.3,159.8,142.5,139.6,139.5,137.8$, $137.2,135.4,132.6,129.1,128.9,128.0,127.4,127.0$, $124.6,122.8,120.3,114.9,113.7,66.1,55.3,54.9$. HRMS 
(ESI) calcd for $\mathrm{C}_{36} \mathrm{H}_{31} \mathrm{~N}_{2} \mathrm{O}_{4} \mathrm{~S}_{2}[\mathrm{M}+\mathrm{H}]^{+}$619.1720, found 619.1720 .

$N$-(2-(4,5-二氢噁唑-2-基)苯基)-2,6-双((4-甲氧基苯 基)硫代)-4-(三氟甲基)苯甲酰胺(3ag): 浅黄色油状液体. $62.3 \mathrm{mg}$, 产率 51\%. ${ }^{1} \mathrm{H}$ NMR (400 $\left.\mathrm{MHz}, \mathrm{CDCl}_{3}\right) \delta: 12.67$ (s, 1H), 8.99 (d, $J=8.4 \mathrm{~Hz}, 1 \mathrm{H}), 7.88$ (d, $J=7.8 \mathrm{~Hz}, 1 \mathrm{H})$, $7.54(\mathrm{t}, J=7.8 \mathrm{~Hz}, 1 \mathrm{H}), 7.41(\mathrm{~d}, J=8.6 \mathrm{~Hz}, 4 \mathrm{H}), 7.15$ (t, $J=7.6 \mathrm{~Hz}, 1 \mathrm{H}), 7.06$ (s, 2H), 6.84 (d, $J=8.6 \mathrm{~Hz}, 4 \mathrm{H}), 4.34$ (t, $J=9.5 \mathrm{~Hz}, 2 \mathrm{H}), 4.02(\mathrm{t}, J=9.4 \mathrm{~Hz}, 2 \mathrm{H}), 3.77(\mathrm{~s}, 6 \mathrm{H})$; ${ }^{13} \mathrm{C}$ NMR (100 MHz, $\left.\mathrm{CDCl}_{3}\right) \delta: 164.7,164.4,160.4,139.7$, $139.3,139.1,136.1,132.7,129.2,123.5,123.1,122.6$, 120.3, 115.2, 113.8, 66.2, 55.3, 54.9. HRMS (ESI) calcd for $\mathrm{C}_{31} \mathrm{H}_{26} \mathrm{~F}_{3} \mathrm{~N}_{2} \mathrm{O}_{4} \mathrm{~S}_{2}[\mathrm{M}+\mathrm{H}]^{+}$611.1281, found 611.1282.

4-溴- $N$-(2-(4,5-二氢噁唑-2-基)苯基)-2,6-双((4-甲氧 基苯基)硫代)苯甲酰胺(3ah)：浅黄色油状液体. $68.4 \mathrm{mg}$, 产率 55\%. ${ }^{1} \mathrm{H}$ NMR (400 MHz, $\left.\mathrm{CDCl}_{3}\right) \delta: 12.59(\mathrm{~s}, 1 \mathrm{H})$, 8.98 (d, $J=8.4 \mathrm{~Hz}, 1 \mathrm{H}), 7.87$ (dd, $J=7.9 \mathrm{~Hz}, 1.3 \mathrm{~Hz}, 1 \mathrm{H})$, $7.55 \sim 7.50(\mathrm{~m}, 1 \mathrm{H}), 7.40(\mathrm{~d}, J=8.7 \mathrm{~Hz}, 4 \mathrm{H}), 7.15 \sim 7.11$ $(\mathrm{m}, 1 \mathrm{H}), 6.93(\mathrm{~s}, 2 \mathrm{H}), 6.84(\mathrm{~d}, J=8.8 \mathrm{~Hz}, 4 \mathrm{H}), 4.03(\mathrm{t}, J=$ $9.5 \mathrm{~Hz}, 2 \mathrm{H}), 4.00(\mathrm{t}, J=9.5 \mathrm{~Hz}, 2 \mathrm{H}), 3.78(\mathrm{~s}, 6 \mathrm{H}) ;{ }^{13} \mathrm{C}$ NMR $\left(100 \mathrm{MHz}, \mathrm{CDCl}_{3}\right) \delta: 165.1,164.4,160.3,139.5$, $139.4,136.1,135.8,132.6,129.4,129.2,123.7,123.0$, $122.9,120.3,115.2,113.7,66.2,55.4,54.9$. HRMS (ESI) calcd for $\mathrm{C}_{30} \mathrm{H}_{26} \mathrm{BrN}_{2} \mathrm{O}_{4} \mathrm{~S}_{2}[\mathrm{M}+\mathrm{H}]^{+}$621.0512, found 621.0512 .

$N$-(2-(4,5-二羟基偶氮基-2-基)苯基)-2,6-双(4-甲氧 基苯基)硫醇)-3-甲基苯甲酰胺(3ai): 浅黄色油状液体. $93.5 \mathrm{mg}$, 产率 84\%. ${ }^{1} \mathrm{H}$ NMR (400 $\left.\mathrm{MHz}, \mathrm{CDCl}_{3}\right) \delta: 12.32$ (s, 1H), 8.99 (dd, $J=8.3 \mathrm{~Hz}, 0.5 \mathrm{~Hz}, 1 \mathrm{H}), 7.81$ (dd, $J=7.9$ $\mathrm{Hz}, 1.4 \mathrm{~Hz}, 1 \mathrm{H}), 7.51 \sim 7.47(\mathrm{~m}, 1 \mathrm{H}), 7.36(\mathrm{~d}, J=8.8 \mathrm{~Hz}$, 2H), $7.15 \sim 7.06(\mathrm{~m}, 5 \mathrm{H}), 6.77(\mathrm{~d}, J=8.8 \mathrm{~Hz}, 2 \mathrm{H}), 6.67$ (d, $J=8.9 \mathrm{~Hz}, 2 \mathrm{H}), 4.26(\mathrm{t}, J=9.5 \mathrm{~Hz}, 2 \mathrm{H}), 3.89$ (t, $J=9.5$ $\mathrm{Hz}, 2 \mathrm{H}), 3.75$ (s, 3H), 3.70 (s, 3H), 2.28 (s, 3H); ${ }^{13} \mathrm{C}$ NMR $\left(100 \mathrm{MHz}, \mathrm{CDCl}_{3}\right) \delta: 166.5,164.2,159.6,158.2,145.2$, $141.9,139.7,134.8,133.1,132.6,132.3,131.8,131.2$, $130.8,129.0,127.4,125.5,122.5,120.2,114.7,114.4$, 113.5, 66.0, 55.3, 55.2, 54.8, 21.1. HRMS (ESI) calcd for $\mathrm{C}_{31} \mathrm{H}_{29} \mathrm{~N}_{2} \mathrm{O}_{4} \mathrm{~S}_{2} \quad[\mathrm{M}+\mathrm{H}]^{+}$557.1563, found 557.1563.

$N$-(2-(4,5-二氢啞唑-2-基)苯基)-3-甲氧基-2,6-双((4甲氧基苯基)硫代)苯甲酰胺(3aj): 浅黄色油状液体. 82.5 $\mathrm{mg}$, 产率 72\%. ${ }^{1} \mathrm{H} \mathrm{NMR}\left(400 \mathrm{MHz}, \mathrm{CDCl}_{3}\right) \delta: 12.23$ (s, $1 \mathrm{H}), 9.00$ (d, $J=8.4 \mathrm{~Hz}, 1 \mathrm{H}), 7.81$ (d, $J=7.8 \mathrm{~Hz}, 1 \mathrm{H}), 7.50$ $(\mathrm{t}, J=7.8 \mathrm{~Hz}, 1 \mathrm{H}), 7.39$ (d, $J=8.6 \mathrm{~Hz}, 1 \mathrm{H}), 7.27$ (t, $J=8.4$ $\mathrm{Hz}, 4 \mathrm{H}), 7.09$ (t, $J=7.5 \mathrm{~Hz}, 1 \mathrm{H}), 6.85$ (d, $J=8.7 \mathrm{~Hz}, 1 \mathrm{H})$, $6.70(\mathrm{~d}, J=8.5 \mathrm{~Hz}, 2 \mathrm{H}), 6.66(\mathrm{~d}, J=8.6 \mathrm{~Hz}, 2 \mathrm{H}), 4.23(\mathrm{t}$,
$J=9.4 \mathrm{~Hz}, 2 \mathrm{H}), 3.79(\mathrm{t}, J=9.4 \mathrm{~Hz}, 2 \mathrm{H}), 3.76(\mathrm{~s}, 3 \mathrm{H}), 3.72$ $(\mathrm{s}, 3 \mathrm{H}), 3.70(\mathrm{~s}, 3 \mathrm{H}) ;{ }^{13} \mathrm{C} \mathrm{NMR}\left(100 \mathrm{MHz}, \mathrm{CDCl}_{3}\right) \delta$ : $165.8,164.1,159.6,159.1,158.5,147.3,139.6,136.1$, $133.5,132.6,132.2,128.9,127.2,127.1,125.3,122.5$, $121.0,120.1,114.5,114.0,113.4,112.7,66.0,56.2,55.3$, 55.2, 54.7. HRMS (ESI) calcd for $\mathrm{C}_{31} \mathrm{H}_{29} \mathrm{~N}_{2} \mathrm{O}_{5} \mathrm{~S}_{2}[\mathrm{M}+\mathrm{H}]^{+}$ 573.1512 , found 573.1512 .

$N$-(2-(4,5-二氢噁唑-2-基)苯基)-2,6-双((4-甲氧基苯 基)硫代)-3-硝基苯甲酰胺(3ak)：黄色油状液体。77.6 $\mathrm{mg}$, 产率 66\%. ${ }^{1} \mathrm{H} \mathrm{NMR}\left(400 \mathrm{MHz}, \mathrm{CDCl}_{3}\right) \delta: 12.55(\mathrm{~s}$, $1 \mathrm{H}), 8.86(\mathrm{~d}, J=8.4 \mathrm{~Hz}, 1 \mathrm{H}), 7.86(\mathrm{dd}, J=7.9 \mathrm{~Hz}, 1.5 \mathrm{~Hz}$, $1 \mathrm{H}), 7.55 \sim 7.50(\mathrm{~m}, 2 \mathrm{H}), 7.43(\mathrm{~d}, J=8.8 \mathrm{~Hz}, 2 \mathrm{H}), 7.23(\mathrm{~d}$, $J=8.8 \mathrm{~Hz}, 2 \mathrm{H}), 7.16 \sim 7.12(\mathrm{~m}, 1 \mathrm{H}), 6.92 \sim 6.90(\mathrm{~m}, 2 \mathrm{H})$, $6.85(\mathrm{~d}, J=8.7 \mathrm{~Hz}, 1 \mathrm{H}), 6.58(\mathrm{~d}, J=8.8 \mathrm{~Hz}, 2 \mathrm{H}), 4.35$ (t, $J=9.6 \mathrm{~Hz}, 2 \mathrm{H}), 4.00(\mathrm{t}, J=9.5 \mathrm{~Hz}, 2 \mathrm{H}), 3.81(\mathrm{~s}, 3 \mathrm{H}), 3.64$ $(\mathrm{s}, 3 \mathrm{H}) ;{ }^{13} \mathrm{C}$ NMR $\left(100 \mathrm{MHz}, \mathrm{CDCl}_{3}\right) \delta$ : 164.2, 163.6, $161.0,159.6,149.6,145.1,140.8,139.2,137.0,134.4$, $132.6,129.7,129.1,127.2,124.8,124.3,123.1,121.0$, $120.2,115.6,114.5,113.6,66.2,55.5,55.1,54.8$. HRMS (ESI) calcd for $\mathrm{C}_{30} \mathrm{H}_{26} \mathrm{~N}_{3} \mathrm{O}_{6} \mathrm{~S}_{2}[\mathrm{M}+\mathrm{H}]^{+}$588.1258, found 588.1258.

3 -溴- $N$-(2-(4,5-二氢噁唑-2-基)苯基)-2,6-双((4-甲氧 基苯基)硫代)苯甲酰胺(3al): 浅黄色油状液体. $75.8 \mathrm{mg}$, 产率 61\%. ${ }^{1} \mathrm{H}$ NMR (400 $\left.\mathrm{MHz}, \mathrm{CDCl}_{3}\right) \delta: 12.39(\mathrm{~s}, 1 \mathrm{H})$, $8.96(\mathrm{~d}, J=8.3 \mathrm{~Hz}, 1 \mathrm{H}), 7.83$ (dd, $J=7.9 \mathrm{~Hz}, 1.3 \mathrm{~Hz}, 1 \mathrm{H})$, $7.53 \sim 7.48(\mathrm{~m}, 2 \mathrm{H}), 7.39(\mathrm{~d}, J=8.8 \mathrm{~Hz}, 2 \mathrm{H}), 7.25(\mathrm{~d}, J=$ $8.8 \mathrm{~Hz}, 2 \mathrm{H}), 7.13 \sim 7.09(\mathrm{~m}, 1 \mathrm{H}), 6.93(\mathrm{~d}, J=8.5 \mathrm{~Hz}, 1 \mathrm{H})$, $6.82(\mathrm{~d}, J=8.8 \mathrm{~Hz}, 2 \mathrm{H}), 6.67(\mathrm{~d}, J=8.8 \mathrm{~Hz}, 2 \mathrm{H}), 4.28$ (t, $J=9.5 \mathrm{~Hz}, 2 \mathrm{H}), 3.90$ (t, $J=9.5 \mathrm{~Hz}, 2 \mathrm{H}), 3.77$ (s, 3H), 3.69 $(\mathrm{s}, 3 \mathrm{H}) ;{ }^{13} \mathrm{C} \mathrm{NMR}\left(100 \mathrm{MHz}, \mathrm{CDCl}_{3}\right) \delta: 165.2,164.2$, $160.2,158.8,144.9,139.4,136.8,135.7,134.4,133.3$, $132.6,132.3,131.9,129.3,129.1,126.3,123.7,122.8$, $120.2,115.1,114.4,113.6,66.1,55.4,55.2,54.8$. HRMS (ESI) calcd for $\mathrm{C}_{30} \mathrm{H}_{26} \mathrm{BrN}_{2} \mathrm{O}_{4} \mathrm{~S}_{2}[\mathrm{M}+\mathrm{H}]^{+}$621.0512, found 621.0512 .

$N$-(2-(4,5-二氢噁唑-2-基)苯基)-2-((4-甲氧基苯基) 硫代)-6-甲基苯甲酰胺(3am): 浅黄色油状液体. $56.9 \mathrm{mg}$, 产率 68\%. ${ }^{1} \mathrm{H}$ NMR (400 MHz, $\left.\mathrm{CDCl}_{3}\right) \delta: 12.37(\mathrm{~s}, 1 \mathrm{H})$, $8.98(\mathrm{~d}, J=8.3 \mathrm{~Hz}, 1 \mathrm{H}), 7.87$ (dd, $J=7.9 \mathrm{~Hz}, 1.4 \mathrm{~Hz}, 1 \mathrm{H})$, $7.54 \sim 7.50(\mathrm{~m}, 1 \mathrm{H}), 7.36(\mathrm{~d}, J=8.8 \mathrm{~Hz}, 2 \mathrm{H}), 7.16 \sim 7.10$ $(\mathrm{m}, 2 \mathrm{H}), 7.06$ (d, $J=7.4 \mathrm{~Hz}, 1 \mathrm{H}), 7.00$ (d, $J=7.8 \mathrm{~Hz}, 1 \mathrm{H})$, $6.78(\mathrm{~d}, \quad J=8.8 \mathrm{~Hz}, 2 \mathrm{H}), 4.29(\mathrm{t}, J=9.5 \mathrm{~Hz}, 2 \mathrm{H}), 3.94(\mathrm{t}$, $J=9.5 \mathrm{~Hz}, 2 \mathrm{H}), 3.75$ (s, 3H), 2.40 (s, 3H); ${ }^{13} \mathrm{C}$ NMR (100 $\left.\mathrm{MHz} \mathrm{CDCl}_{3}\right) \delta: 167.6,164.3,159.6,139.6,139.0,135.4$, $135.3,135.0,132.6,129.2,129.1,128.5,128.4,125.3$, 
122.8, 120.2, 114.7, 113.6, 66.1, 55.3, 54.8, 19.6. HRMS (ESI) calcd for $\mathrm{C}_{24} \mathrm{H}_{23} \mathrm{~N}_{2} \mathrm{O}_{3} \mathrm{~S}[\mathrm{M}+\mathrm{H}]^{+}$419.1424, found 419.1423.

$N$-(2-(4,5-二氢噁唑-2-基)苯基)-2-甲氧基-6-((4-甲氧 基苯基)硫代)苯甲酰胺(3an): 浅黄色油状液体. $37.4 \mathrm{mg}$, 产率 $43 \%$. ${ }^{1} \mathrm{H}$ NMR (400 $\left.\mathrm{MHz}, \mathrm{CDCl}_{3}\right) \delta: 12.35(\mathrm{~s}, 1 \mathrm{H})$, 8.95 (d, $J=8.4 \mathrm{~Hz}, 1 \mathrm{H}), 7.80$ (d, $J=7.8 \mathrm{~Hz}, 1 \mathrm{H}), 7.44$ (t, $J=7.7 \mathrm{~Hz}, 1 \mathrm{H}), 7.34(\mathrm{~d}, J=8.4 \mathrm{~Hz}, 2 \mathrm{H}), 7.10$ (t, $J=8.1$ $\mathrm{Hz}, 1 \mathrm{H}), 7.03$ (t, $J=7.5 \mathrm{~Hz}, 1 \mathrm{H}), 6.75$ (d, $J=8.4 \mathrm{~Hz}, 2 \mathrm{H})$, $6.69(\mathrm{~d}, J=8.3 \mathrm{~Hz}, 1 \mathrm{H}), 6.59(\mathrm{~d}, J=8.0 \mathrm{~Hz}, 1 \mathrm{H}), 4.25$ (t, $J=9.4 \mathrm{~Hz}, 2 \mathrm{H}), 3.93$ (t, $J=9.4 \mathrm{~Hz}, 2 \mathrm{H}), 3.74$ (s, 3H), 3.71 $(\mathrm{s}, 3 \mathrm{H}) ;{ }^{13} \mathrm{C}$ NMR $\left(100 \mathrm{MHz}, \mathrm{CDCl}_{3}\right) \delta: 165.2,164.3$, $159.8,156.7,139.9,138.5,135.8,132.6,130.1,129.1$, $127.4,124.6,122.5,122.0,120.3,114.8,113.5,108.7$, 66.0, 56.0, 55.3, 54.9. HRMS (ESI) calcd for $\mathrm{C}_{24} \mathrm{H}_{23} \mathrm{~N}_{2} \mathrm{O}_{4} \mathrm{~S}$ $[\mathrm{M}+\mathrm{H}]^{+}$435.1373, found 435.1373 .

$N$-(2-(4,5-二氢噁唑-2-基)苯基)-2-((4-甲氧基苯基) 硫代)-6-硝基苯甲酰胺(3ao): 黄色油状液体. $28.8 \mathrm{mg}$, 产率 32\%. ${ }^{1} \mathrm{H}$ NMR (400 $\mathrm{MHz}, \mathrm{CDCl}_{3}$ ) $\delta: 12.69$ (s, 1H), $8.87(\mathrm{~d}, J=7.8 \mathrm{~Hz}, 1 \mathrm{H}), 7.93 \sim 7.87(\mathrm{~m}, 2 \mathrm{H}), 7.57 \sim 7.53$ (m, 1H), 7.44 (d, $J=8.8 \mathrm{~Hz}, 2 \mathrm{H}), 7.35$ (t, $J=8.0 \mathrm{~Hz}, 1 \mathrm{H})$, $7.29 \sim 7.26(\mathrm{~m}, 1 \mathrm{H}), 7.17 \sim 7.13(\mathrm{~m}, 1 \mathrm{H}), 6.89(\mathrm{~d}, J=8.8$ $\mathrm{Hz}, 2 \mathrm{H}), 4.34$ (t, $J=9.5 \mathrm{~Hz}, 2 \mathrm{H}), 3.98(\mathrm{t}, J=9.6 \mathrm{~Hz}, 2 \mathrm{H})$, $3.81(\mathrm{~s}, 3 \mathrm{H}) ;{ }^{13} \mathrm{C} \mathrm{NMR}\left(100 \mathrm{MHz}, \mathrm{CDCl}_{3}\right) \delta: 164.6,163.3$, $160.6,146.4,141.2,139.3,136.5,134.3,132.7,131.7$, $129.6,129.2,123.1,122.3,121.6,120.5,115.4,113.7$, 66.2, 55.4, 54.6. HRMS (ESI) calcd for $\mathrm{C}_{23} \mathrm{H}_{20} \mathrm{~N}_{3} \mathrm{O}_{5} \mathrm{~S}[\mathrm{M}$ $+\mathrm{H}]^{+} 450.1118$, found 450.1117 .

$N$-(2-(4,5-二氢噁唑-2-基)苯基)-2,6-双(对甲苯基硫) 苯甲酰胺(3ba): 浅黄色油状液体. $71.5 \mathrm{mg}$, 产率 $70 \%$. ${ }^{1} \mathrm{H}$ NMR (400 MHz, $\mathrm{CDCl}_{3}$ ) $\delta: 11.10$ (s, 1H), 8.68 (d, $J=$ $8.4 \mathrm{~Hz}, 1 \mathrm{H}), 7.46 \sim 7.43(\mathrm{~m}, 2 \mathrm{H}), 7.27$ (d, $J=7.8 \mathrm{~Hz}, 4 \mathrm{H})$, $7.04 \sim 7.02(\mathrm{~m}, 5 \mathrm{H}), 6.86(\mathrm{~d}, J=7.8 \mathrm{~Hz}, 2 \mathrm{H}), 6.67(\mathrm{~s}, 1 \mathrm{H})$, $3.61(\mathrm{t}, J=5.5 \mathrm{~Hz}, 2 \mathrm{H}), 3.55(\mathrm{t}, J=5.2 \mathrm{~Hz}, 2 \mathrm{H}), 2.24(\mathrm{~s}$, $6 \mathrm{H}) ;{ }^{13} \mathrm{C}$ NMR (100 MHz, $\left.\mathrm{CDCl}_{3}\right) \delta: 167.9,164.7,137.9$, $137.5,137.1,135.2,132.2,131.8,129.4,129.1,128.9$, $127.7,125.6,122.5,121.3,119.9,42.3,40.5,20.1$. HRMS (ESI) calcd for $\mathrm{C}_{30} \mathrm{H}_{27} \mathrm{~N}_{2} \mathrm{O}_{2} \mathrm{~S}_{2}[\mathrm{M}+\mathrm{H}]^{+}$511.1508, found 511.1509 .

$N$-(2-(4,5-二氢噁唑-2-基)苯基)-2,6-双((4-异丙基苯 基)硫代)苯甲酰胺(3bb): 浅黄色油状液体. $69.2 \mathrm{mg}$, 产 率 $61 \% .{ }^{1} \mathrm{H}$ NMR (400 MHz, $\mathrm{CDCl}_{3}$ ) $\delta: 12.51$ (s, 1H), 8.95 $(\mathrm{d}, J=8.4 \mathrm{~Hz}, 1 \mathrm{H}), 7.84$ (d, $J=7.9 \mathrm{~Hz}, 1 \mathrm{H}), 7.49$ (t, $J=7.8$ $\mathrm{Hz}, 1 \mathrm{H}), 7.33(\mathrm{~d}, J=8.1 \mathrm{~Hz}, 4 \mathrm{H}), 7.13 \sim 7.06(\mathrm{~m}, 8 \mathrm{H})$, $4.30(\mathrm{t}, J=9.5 \mathrm{~Hz}, 2 \mathrm{H}), 3.98(\mathrm{t}, J=9.5 \mathrm{~Hz}, 2 \mathrm{H}), 2.88 \sim$ $2.81(\mathrm{~m}, 2 \mathrm{H}), 1.20(\mathrm{~d}, J=6.9 \mathrm{~Hz}, 12 \mathrm{H}) ;{ }^{13} \mathrm{C}$ NMR $(100$ $\left.\mathrm{MHz}, \mathrm{CDCl}_{3}\right) \delta: 165.9,164.3,148.7,140.3,139.6,135.7$, $132.7,132.6,131.5,129.8,129.7,129.1,127.4,122.7$, 120.3, 113.6, 66.1, 54.8, 33.8, 23.9. HRMS (ESI) calcd for $\mathrm{C}_{34} \mathrm{H}_{35} \mathrm{~N}_{2} \mathrm{O}_{2} \mathrm{~S}_{2}[\mathrm{M}+\mathrm{H}]^{+}$567.2134, found 567.2135

2,6-双((4-(叔丁基)苯基)硫代)- $N$-(2-(4,5-二氢噁唑2-基)苯基)苯甲酰胺(3bc): 浅黄色油状液体. $74.9 \mathrm{mg}$, 产率 63\%. ${ }^{1} \mathrm{H}$ NMR (400 MHz, $\left.\mathrm{CDCl}_{3}\right) \delta: 12.51(\mathrm{~s}, 1 \mathrm{H})$, $8.94(\mathrm{~d}, J=8.4 \mathrm{~Hz}, 1 \mathrm{H}), 7.84(\mathrm{~d}, J=7.9 \mathrm{~Hz}, 1 \mathrm{H}), 7.49$ (t, $J=7.8 \mathrm{~Hz}, 1 \mathrm{H}), 7.34(\mathrm{~d}, J=8.4 \mathrm{~Hz}, 4 \mathrm{H}), 7.27$ (d, $J=8.4$ $\mathrm{Hz}, 4 \mathrm{H}), 7.15 \sim 7.07(\mathrm{~m}, 4 \mathrm{H}), 4.30(\mathrm{t}, J=9.5 \mathrm{~Hz}, 2 \mathrm{H}), 3.98$ $(\mathrm{t}, J=9.5 \mathrm{~Hz}, 2 \mathrm{H}), 1.27(\mathrm{~s}, 18 \mathrm{H}) ;{ }^{13} \mathrm{C} \mathrm{NMR}(100 \mathrm{MHz}$, $\left.\mathrm{CDCl}_{3}\right) \delta: 165.9,164.3,150.9,140.5,139.6,135.6,132.6$, $132.2,131.3,130.0,129.8,129.1,126.2,122.7,120.3$, 113.6, 66.1, 54.8, 34.6, 31.3. HRMS (ESI) calcd for $\mathrm{C}_{36} \mathrm{H}_{39} \mathrm{~N}_{2} \mathrm{O}_{2} \mathrm{~S}_{2}[\mathrm{M}+\mathrm{H}]^{+}$595.2447, found 595.2448.

$N$-(2-(4,5-二氢噁唑-2-基)苯基)-2,6-双((4-氟苯基)硫 代)苯甲酰胺(3bd): 浅黄色油状液体. $49.8 \mathrm{mg}$, 产率 $48 \%$. ${ }^{1} \mathrm{H}$ NMR (400 MHz, $\mathrm{CDCl}_{3}$ ) $\delta: 12.47$ (s, 1H), 8.95 $(\mathrm{d}, J=8.4 \mathrm{~Hz}, 1 \mathrm{H}), 7.86(\mathrm{~d}, J=7.0 \mathrm{~Hz}, 1 \mathrm{H}), 7.52$ (t, $J=7.5$ $\mathrm{Hz}, 1 \mathrm{H}), 7.38$ (dd, $J=8.6 \mathrm{~Hz}, 5.4 \mathrm{~Hz}, 4 \mathrm{H}), 7.19 \sim 7.09$ (m, $4 \mathrm{H}), 6.95$ (t, $J=8.6 \mathrm{~Hz}, 4 \mathrm{H}), 4.32$ (t, $J=9.5 \mathrm{~Hz}, 2 \mathrm{H}), 3.94$ $(\mathrm{t}, J=9.5 \mathrm{~Hz}, 2 \mathrm{H}) ;{ }^{13} \mathrm{C} \mathrm{NMR}\left(100 \mathrm{MHz}, \mathrm{CDCl}_{3}\right) \delta: 165.6$, $164.4,163.8,161.3,140.9,139.4,135.4,134.8,134.7$, $132.7,130.6,130.0,129.9,129.2,122.9,120.2,116.5$, 116.2, 113.6, 66.1, 54.8. HRMS (ESI) calcd for $\mathrm{C}_{28} \mathrm{H}_{21} \mathrm{~F}_{2} \mathrm{~N}_{2} \mathrm{O}_{2} \mathrm{~S}_{2}[\mathrm{M}+\mathrm{H}]^{+}$519.1007, found 519.1006.

$N$-(2-(4,5-二氢噁唑-2-基)苯基)-2,6-双((3-甲氧基苯 基)硫代)苯甲酰胺(3be): 浅黄色油状液体. $86.8 \mathrm{mg}$, 产 率 $80 \% .{ }^{1} \mathrm{H} \mathrm{NMR}\left(400 \mathrm{MHz}, \mathrm{CDCl}_{3}\right) \delta: 12.49$ (s, 1H), 8.94 (d, $J=8.2 \mathrm{~Hz}, 1 \mathrm{H}), 7.82(\mathrm{dd}, J=7.9 \mathrm{~Hz}, 1.4 \mathrm{~Hz}, 1 \mathrm{H})$, $7.51 \sim 7.47(\mathrm{~m}, 1 \mathrm{H}), 7.26 \sim 7.20(\mathrm{~m}, 3 \mathrm{H}), 7.16 \sim 7.07(\mathrm{~m}$, $3 \mathrm{H}), 6.95 \sim 6.90(\mathrm{~m}, 4 \mathrm{H}), 6.73(\mathrm{dd}, J=8.2 \mathrm{~Hz}, 2.0 \mathrm{~Hz}$, $2 \mathrm{H}), 4.26(\mathrm{t}, J=9.5 \mathrm{~Hz}, 2 \mathrm{H}), 3.91(\mathrm{t}, J=9.5 \mathrm{~Hz}, 2 \mathrm{H}), 3.68$ $(\mathrm{s}, 6 \mathrm{H}) ;{ }^{13} \mathrm{C} \mathrm{NMR}\left(100 \mathrm{MHz}, \mathrm{CDCl}_{3}\right) \delta: 165.7,164.2$, $159.9,142.4,139.5,136.6,134.4,132.5,131.9,130.0$, $129.9,129.1,124.0,122.7,120.2,117.0,113.6,113.2$, 66.1, 55.3, 54.6. HRMS (ESI) calcd for $\mathrm{C}_{30} \mathrm{H}_{27} \mathrm{~N}_{2} \mathrm{O}_{4} \mathrm{~S}_{2}[\mathrm{M}$ $+\mathrm{H}]^{+}$543.1407, found 543.1404.

$N$-(2-(4,5-二羟基偶氮基-2-基)苯基)-2,6-双(2-甲氧 基苯基)硫醇)苯甲酰胺(3bf): 浅黄色油状液体. $76.0 \mathrm{mg}$, 产率 70\%. ${ }^{1} \mathrm{H} \mathrm{NMR}\left(400 \mathrm{MHz}, \mathrm{CDCl}_{3}\right) \delta: 12.42(\mathrm{~s}, 1 \mathrm{H})$, $8.93(\mathrm{~d}, J=8.3 \mathrm{~Hz}, 1 \mathrm{H}), 7.81(\mathrm{dd}, J=7.9 \mathrm{~Hz}, 1.4 \mathrm{~Hz}, 1 \mathrm{H})$, $7.49 \sim 7.44(\mathrm{~m}, 1 \mathrm{H}), 7.21 \sim 7.17(\mathrm{~m}, 7 \mathrm{H}), 7.09 \sim 7.05(\mathrm{~m}$, $1 \mathrm{H}), 6.85 \sim 6.80(\mathrm{~m}, 4 \mathrm{H}), 4.27(\mathrm{t}, J=9.5 \mathrm{~Hz}, 2 \mathrm{H}), 3.92(\mathrm{t}$, 
$J=9.5 \mathrm{~Hz}, 2 \mathrm{H}), 3.74(\mathrm{~s}, 6 \mathrm{H}) ;{ }^{13} \mathrm{C} \mathrm{NMR}\left(100 \mathrm{MHz}, \mathrm{CDCl}_{3}\right)$ $\delta: 165.7,164.1,157.7,143.0,139.7,133.8,132.6,132.4$, $131.8,129.7,128.9,128.5,124.1,122.5,121.1,120.4$, 113.6, 110.9, 66.0, 55.8, 54.7. HRMS (ESI) calcd for $\mathrm{C}_{30} \mathrm{H}_{27} \mathrm{~N}_{2} \mathrm{O}_{4} \mathrm{~S}_{2}[\mathrm{M}+\mathrm{H}]^{+}$543.1407, found 543.1403.

$N$-(2-(4,5-二氢噁唑-2-基)苯基)-2,6-双(邻甲苯硫基) 苯甲酰胺 $(3 \mathrm{bg})$ : 浅黄色油状液体. $69.5 \mathrm{mg}$, 产率 $68 \%$. ${ }^{1} \mathrm{H}$ NMR (400 MHz, $\left.\mathrm{CDCl}_{3}\right) \delta: 12.50(\mathrm{~s}, 1 \mathrm{H}), 8.89$ (d, $J=$ $8.4 \mathrm{~Hz}, 1 \mathrm{H}), 7.77$ (dd, $J=7.9 \mathrm{~Hz}, 1.4 \mathrm{~Hz}, 1 \mathrm{H}), 7.45 \sim 7.41$ $(\mathrm{m}, 1 \mathrm{H}), 7.31(\mathrm{~d}, J=7.5 \mathrm{~Hz}, 2 \mathrm{H}), 7.11 \sim 6.96(\mathrm{~m}, 8 \mathrm{H}), 6.77$ $(\mathrm{d}, J=7.9 \mathrm{~Hz}, 2 \mathrm{H}), 4.23(\mathrm{t}, J=9.5 \mathrm{~Hz}, 2 \mathrm{H}), 3.88(\mathrm{t}, J=9.5$ $\mathrm{Hz}, 2 \mathrm{H}), 2.24(\mathrm{~s}, 6 \mathrm{H}) ;{ }^{13} \mathrm{C} \mathrm{NMR}\left(100 \mathrm{MHz}, \mathrm{CDCl}_{3}\right) \delta$ : $164.7,163.2,139.6,138.6,138.3,134.4,133.0,132.2$, $131.5,129.6,128.7,128.0,127.2,127.1,125.6,121.7$, 119.2, 112.5, 65.0, 53.7, 19.6. HRMS (ESI) calcd for $\mathrm{C}_{30} \mathrm{H}_{27} \mathrm{~N}_{2} \mathrm{O}_{2} \mathrm{~S}_{2}[\mathrm{M}+\mathrm{H}]^{+}$511.1508, found 511.1505.

$N$-(2-(4,5-二氢噁唑-2-基)苯基)-3,5-双((4-甲氧基苯 基)硫代)异烟酰胺(5a): 浅黄色油状液体. $52.2 \mathrm{mg}$, 产率 $48 \%$. ${ }^{1} \mathrm{H}$ NMR (400 MHz, $\mathrm{CDCl}_{3}$ ) $\delta: 12.52$ (s, 1H), 8.85 (d, $J=8.4 \mathrm{~Hz}, 1 \mathrm{H}), 8.08$ (s, 2H), 7.80 (dd, $J=7.8 \mathrm{~Hz}, 1.3$ $\mathrm{Hz}, 1 \mathrm{H}), 7.46$ (t, $J=7.8 \mathrm{~Hz}, 1 \mathrm{H}), 7.32$ (d, $J=8.8 \mathrm{~Hz}, 4 \mathrm{H})$, $7.08(\mathrm{t}, J=7.6 \mathrm{~Hz}, 1 \mathrm{H}), 6.71(\mathrm{~d}, J=8.8 \mathrm{~Hz}, 4 \mathrm{H}), 4.27(\mathrm{t}$, $J=9.5 \mathrm{~Hz}, 2 \mathrm{H}), 3.89(\mathrm{t}, J=9.5 \mathrm{~Hz}, 2 \mathrm{H}), 3.68(\mathrm{~s}, 6 \mathrm{H}) ;{ }^{13} \mathrm{C}$ NMR $\left(100 \mathrm{MHz}, \mathrm{CDCl}_{3}\right) \delta: 163.3,162.4,159.2,147.7$, $143.5,138.0,134.7,131.6,131.4,128.1,122.1,121.8$, 119.2, 114.0, 112.7, 65.2, 54.3, 53.7. HRMS (ESI) calcd for $\mathrm{C}_{29} \mathrm{H}_{26} \mathrm{~N}_{3} \mathrm{O}_{4} \mathrm{~S}_{2}[\mathrm{M}+\mathrm{H}]^{+}$544.1359, found 544.1363.

$N$-(2-(4,5-二氢噁唑-2-基)苯基)-3,5-双((3-甲氧基苯 基)硫代)异烟酰胺(5b): 浅黄色油状液体. $51.1 \mathrm{mg}$, 产率 47\%. ${ }^{1} \mathrm{H}$ NMR (400 MHz, $\mathrm{CDCl}_{3}$ ) $\delta: 12.53$ (s, 1H), 8.79 (d, $J=8.4 \mathrm{~Hz}, 1 \mathrm{H}), 8.25$ (s, 2H), $7.76(\mathrm{~d}, J=7.8 \mathrm{~Hz}, 1 \mathrm{H})$, $7.41(\mathrm{t}, J=7.8 \mathrm{~Hz}, 1 \mathrm{H}), 7.19 \sim 7.13(\mathrm{~m}, 4 \mathrm{H}), 7.04(\mathrm{t}, J=$ $7.6 \mathrm{~Hz}, 1 \mathrm{H}), 6.77 \sim 6.73(\mathrm{~m}, 4 \mathrm{H}), 4.23(\mathrm{t}, J=9.5 \mathrm{~Hz}, 2 \mathrm{H})$, $3.85(\mathrm{t}, J=9.5 \mathrm{~Hz}, 2 \mathrm{H}), 3.66(\mathrm{~s}, 6 \mathrm{H}) ;{ }^{13} \mathrm{C}$ NMR (100 MHz, $\left.\mathrm{CDCl}_{3}\right) \delta: 164.3,163.3,158.2,150.8,147.5,139.2,133.7$, $132.5,130.1,129.7,129.0,123.0,121.6,121.2,120.4$, 113.7, 111.1, 66.1, 55.7, 54.7. HRMS (ESI) calcd for $\mathrm{C}_{29} \mathrm{H}_{26} \mathrm{~N}_{3} \mathrm{O}_{4} \mathrm{~S}_{2}[\mathrm{M}+\mathrm{H}]^{+}$544.1359, found 544.1359.

$N$-(2-(4,5-二氢噁唑-2-基)苯基)-3,5-双((2-甲氧基苯 基)硫代)异烟酰胺(5c): 浅黄色油状液体. $40.2 \mathrm{mg}$, 产率 37\%. ${ }^{1} \mathrm{H}$ NMR (400 MHz, $\mathrm{CDCl}_{3}$ ) $\delta: 12.65$ (s, 1H), 8.87 $(\mathrm{d}, J=8.4 \mathrm{~Hz}, 1 \mathrm{H}), 8.41$ (s, 2H), 7.84 (dd, $J=7.8 \mathrm{~Hz}, 1.2$ $\mathrm{Hz}, 1 \mathrm{H}), 7.52 \sim 7.48(\mathrm{~m}, 1 \mathrm{H}), 7.18 \sim 7.11(\mathrm{~m}, 3 \mathrm{H}), 6.98 \sim$ $6.93(\mathrm{~m}, 4 \mathrm{H}), 6.76(\mathrm{dd}, J=8.2 \mathrm{~Hz}, 2.2 \mathrm{~Hz}, 2 \mathrm{H}), 4.30(\mathrm{t}$, $J=9.5 \mathrm{~Hz}, 2 \mathrm{H}), 3.92(\mathrm{t}, J=9.5 \mathrm{~Hz}, 2 \mathrm{H}), 3.68(\mathrm{~s}, 6 \mathrm{H}) ;{ }^{13} \mathrm{C}$
NMR (100 MHz, $\left.\mathrm{CDCl}_{3}\right) \delta: 164.3,163.3,160.1,151.4$, $147.7,139.0,134.7,132.6,130.6,130.1,129.1,124.6$, 123.2, 120.2, 117.6, 113.9, 113.7, 66.2, 55.3, 54.5. HRMS (ESI) calcd for $\mathrm{C}_{29} \mathrm{H}_{26} \mathrm{~N}_{3} \mathrm{O}_{4} \mathrm{~S}_{2}[\mathrm{M}+\mathrm{H}]^{+}$544.1359, found 544.1365 .

$N$-(2-(4,5-二氢噁唑-2-基)苯基)-3,5-双((4-异丙基苯 基)硫代)异烟酰胺(5d): 浅黄色油状液体. $50.0 \mathrm{mg}$, 产率 $44 \%$. ${ }^{1} \mathrm{H}$ NMR (400 MHz, $\mathrm{CDCl}_{3}$ ) $\delta: 12.70$ (s, 1H), 8.88 $(\mathrm{d}, J=8.3 \mathrm{~Hz}, 1 \mathrm{H}), 8.23$ (s, 2H), 7.87 (dd, $J=7.9 \mathrm{~Hz}, 1.2$ $\mathrm{Hz}, 1 \mathrm{H}), 7.54 \sim 7.49(\mathrm{~m}, 1 \mathrm{H}), 7.37(\mathrm{~d}, J=8.2 \mathrm{~Hz}, 4 \mathrm{H})$, $7.14(\mathrm{~d}, J=8.2 \mathrm{~Hz}, 5 \mathrm{H}), 4.34$ (t, $J=9.5 \mathrm{~Hz}, 2 \mathrm{H}), 4.00$ (t, $J=9.5 \mathrm{~Hz}, 2 \mathrm{H}), 2.90 \sim 2.80(\mathrm{~m}, 2 \mathrm{H}), 1.20(\mathrm{~d}, J=6.9 \mathrm{~Hz}$, $12 \mathrm{H}) ;{ }^{13} \mathrm{C}$ NMR $\left(100 \mathrm{MHz}, \mathrm{CDCl}_{3}\right) \delta: 164.4,163.5,149.6$, $149.5,145.5,139.1,133.2,132.7,131.7,129.7,129.2$, $127.7,123.2,120.3,113.7,66.2,54.7,33.8,23.8$. HRMS (ESI) calcd for $\mathrm{C}_{33} \mathrm{H}_{34} \mathrm{~N}_{3} \mathrm{O}_{2} \mathrm{~S}_{2}[\mathrm{M}+\mathrm{H}]^{+}$568.2087, found 568.2087 .

3,5 -双((4-(叔丁基)苯基)硫代)- $N$-(2-(4,5-二氢啞唑2-基)苯基)异烟酰胺(5e): 浅黄色油状液体. $54.8 \mathrm{mg}$, 产 率 46\%. ${ }^{1} \mathrm{H}$ NMR $\left(400 \mathrm{MHz}, \mathrm{CDCl}_{3}\right) \delta: 12.71(\mathrm{~s}, 1 \mathrm{H}), 8.88$ (d, $J=8.4 \mathrm{~Hz}, 1 \mathrm{H}), 8.24$ (s, 2H), 7.86 (dd, $J=7.9 \mathrm{~Hz}, 1.2$ $\mathrm{Hz}, 1 \mathrm{H}), 7.53 \sim 7.49(\mathrm{~m}, 1 \mathrm{H}), 7.37(\mathrm{~d}, J=8.4 \mathrm{~Hz}, 4 \mathrm{H})$, 7.29 (d, $J=8.4 \mathrm{~Hz}, 4 \mathrm{H}), 7.13$ (t, $J=7.6 \mathrm{~Hz}, 1 \mathrm{H}), 4.33$ (t, $J=9.5 \mathrm{~Hz}, 2 \mathrm{H}), 4.00(\mathrm{t}, J=9.5 \mathrm{~Hz}, 2 \mathrm{H}), 1.27(\mathrm{~s}, 18 \mathrm{H}) ;{ }^{13} \mathrm{C}$ NMR (100 MHz, $\left.\mathrm{CDCl}_{3}\right) \delta: 164.4,163.5,151.7,149.7$, $145.7,139.1,132.8,132.7,131.6,129.4,129.2,126.6$, 123.2, 120.3, 113.7, 66.2, 54.8, 34.6, 31.2. HRMS (ESI) calcd for $\mathrm{C}_{35} \mathrm{H}_{38} \mathrm{~N}_{3} \mathrm{O}_{2} \mathrm{~S}_{2}[\mathrm{M}+\mathrm{H}]{ }^{+}$596.2400, found 596.2401 .

3,5 -双((4-溴苯基)硫代)- $N$-(2-(4,5-二氢噁唑-2-基)苯 基)异烟酰胺(5f): 浅黄色油状液体. $78.3 \mathrm{mg}$, 产率 $61 \%$. ${ }^{1} \mathrm{H}$ NMR (400 MHz, $\mathrm{CDCl}_{3}$ ) $\delta: 12.43$ (s, 1H), 8.76 (d, $J=$ $8.0 \mathrm{~Hz}, 1 \mathrm{H}), 8.39$ (s, 2H), 7.79 (dd, $J=7.9 \mathrm{~Hz}, 1.3 \mathrm{~Hz}$, $1 \mathrm{H}), 7.47 \sim 7.42(\mathrm{~m}, 1 \mathrm{H}), 7.25(\mathrm{~d}, J=8.5 \mathrm{~Hz}, 4 \mathrm{H}), 7.14(\mathrm{~d}$, $J=8.5 \mathrm{~Hz}, 4 \mathrm{H}), 7.10 \sim 7.06(\mathrm{~m}, 1 \mathrm{H}), 4.27(\mathrm{t}, J=9.6 \mathrm{~Hz}$, $2 \mathrm{H}), 3.79$ (t, $J=9.6 \mathrm{~Hz}, 2 \mathrm{H}) ;{ }^{13} \mathrm{C} \mathrm{NMR}\left(100 \mathrm{MHz}, \mathrm{CDCl}_{3}\right.$ ) $\delta: 163.4,161.8,151.1,147.7,137.7,132.6,131.9,131.7$, 131.4, 129.1, 128.3, 122.4, 121.4, 119.0, 112.5, 65.2, 53.6. HRMS (ESI) calcd for $\mathrm{C}_{27} \mathrm{H}_{20} \mathrm{Br}_{2} \mathrm{~N}_{3} \mathrm{O}_{2} \mathrm{~S}_{2} \quad[\mathrm{M}+\mathrm{H}]{ }^{+}$ 639.9358 , found 639.9356 .

辅助材料(Supporting Information) 化合物 3aa 的单晶 数据、化合物 $\mathbf{3}$ 和 $\mathbf{5}$ 的核磁图谱. 这些材料可以免费从 本刊网站(http://sioc-journal.cn/)上下载. 


\section{References}

[1] Fraústo da Silva, J. R.; Williams, R. J. P. The Biological Chemistry of the Elements, Oxford University Press, New York, 2001

[2] (a) Scott, K. A.; Njardarson, J. T. Top. Curr. Chem. 2018, 376, 5. (b) Zhao, J.; Jiang, X. Chin. Chem. Lett. 2018, 29, 1079.

[3] Mellah, M.; Voituriez, A.; Schulz, E. Chem. Rev. 2007, 107, 5133.

[4] Cinar, M. E.; Ozturk, T. Chem. Rev. 2015, 115, 3036.

[5] (a) Khanal, H. D.; Kim, S. H. Lee, Y. R. RSC Adv. 2016, 6, 58501. (b) Dong, Y.-T.; Jin, Q.; Zhou, L.; Chen, J. Org. Lett. 2016, 18, 5708 .

(c) Li, Y.; Zhu, F.; Wang, Z.; Wu, X.-F. Chem. Asian J. 2016, 11, 3503 .

(d) Yang, Z.-H.; An, Y.-L.; Chen, Y.; Shao, Z.-Y.; Zhao, S.-Y. Adv. Synth. Catal. 2016, 358, 3869.

(e) Shen, C.; Zhang, P.; Sun, Q.; Bai, S.; Hor, T. S. A.; Liu, X. Chem. Soc. Rev. 2015, 44, 291.

[6] For selected reviews on transition metals catalyzed $\mathrm{C}-\mathrm{H}$ functionalization, see:

(a) Liu, Y.-H.; Xia, Y.-N.; Shi, B.-F. Chin. J. Chem. 2020, 38, 635.

(b) Shang, M.; Sun, S.-Z.; Wang, H.-L.; Wang, M.-M.; Dai, H.-X. Synthesis 2016, 48, 4381.

(c) Rao, W.-H.; Shi, B.-F. Org. Chem. Front. 2016, 3, 1028.

(d) Liu, J.; Chen, G.; Tan, Z. Adv. Synth. Catal. 2016, 358, 1174.

(e) Hu, Y.; Wang, C. Acta Phys.-Chim. Sin. 2019, 35, 913.

[7] (a) Jiang, Y.-J.; Liang, G.-H.; Zhang, C.; Loh, T.-P. Eur. J. Org. Chem. 2016, 2016, 3326.

(b) Iwasaki, M.; Nishihara, Y. Dalton Trans 2016, 45, 15278.

(c) Iwasaki, M.; Kaneshika, W.; Tsuchiya, Y.; Nakajima, K.; Nishihara, Y. J. Org. Chem. 2014, 79, 11330.

(d) Iwasaki, M.; Lyanaga, M.; Tsuchiya, Y.; Nishimura, Y.; Li, W.-J.; Li, Z.-P.; Nishihara, Y. Chem.-Eur. J. 2014, 20, 2459.

[8] (a) Xie, W.-C.; Li, B.; Wang, B.-Q. J. Org. Chem. 2016, 81, 396. (b) Yang, Y.-X.; Hou, W.; Qin, L.-H.; Du, J.-J.; Feng, H.-J.; Zhou, B.; Li, Y.-C. Chem.-Eur. J. 2014, 20, 416.

[9] (a) Ma, W.; Weng, Z.; Fang, X.; Gu, L.; Song, Y.; Ackermann, L. Eur. J. Org. Chem. 2019, 2019, 41.

(b) Ma, W.; Weng, Z.; Rogge, T.; Gu, Li.; Lin, J.; Peng, A.; Luo, X.; Gou, X.; Ackermann, L. Adv. Synth. Catal. 2018, 360, 704.

(c) Mandal, A.; Dana, S.; Sahoo, H.; Grandhi, G. S.; Baidya, M.
Org. Lett. 2017, 19, 2430.

(d) Ma, W.; Dong, H.; Wang, D.; Ackermann, L. Adv. Synth. Catal. 2017, 359, 966.

[10] (a) Gao, F.; Zhu, W.; Zhang, D.-Y.; Li, S.-J.; Wang, J.; Liu, H. J. Org. Chem. 2016, 81, 9122.

(b) Yan, S.-Y.; Liu, Y.-J.; Liu, B.; Liu, Y.-H.; Shi, B.-F. Chem. Commun. 2015, 51, 4069.

(c) Yan, S.-Y.; Liu, Y.-J.; Liu, B.; Liu, Y.-H.; Zhang, Z.-Z.; Shi, B.-F. Chem. Commun. 2015, 51, 7341.

(d) Lin, C.; Yu, W.-L.; Yao, J.-Z.; Wang, B.-J.; Liu, Z.-X.; Zhang, Y.-H. Org. Lett. 2015, 17, 1340.

(e) Wang, X.; Qiu, R.-H.; Yan, C.-Y.; Reddy, V. P.; Zhu, L.-Z.; Xu, X.-H.; Yin, S.-F. Org. Lett. 2015, 17, 1970.

[11] Chen, X.; Hao, X.-S.; Goodhue, C. E.; Yu, J.-Q. J. Am. Chem. Soc. 2006, 128, 6790 .

[12] Chu, L.-L.; Yue, X.-Y.; Qing, F.-L. Org. Lett. 2010, 12, 1644.

[13] Tran, L. D.; Popov, I.; Daugulis, O. J. Am. Chem. Soc. 2012, 134, 18237.

[14] Liu, S.-L.; Li, X.-H.; Shi, T.-H.; Yang, G.-C.; Wang, H.-L.; Gong, J.-F.; Song, M.- P. Eur. J. Org. Chem. 2017, 2017, 2280.

[15] (a) Li, Y.; Liu, Y.-J.; Shi, B.-F. Adv. Synth. Catal. 2017, 359, 4117.

(b) Rao, W.-H.; Shi, B.-F. Org. Lett. 2015, 17, 2784.

(c) Chen, F.-J.; Liao, G.; Li, X.; Wu, J.; Shi, B.-F. Org. Lett. 2014, 16, 5644.

[16] (a) Jiang, Y.; Feng, Y.-Y.; Zou, J.-X.; Lei, S.; Hu, X.-L.; Yin, G.-F.; Tan, W.; Wang, Z. J. Org. Chem. 2019, 84, 10490.

(b) Kong, W.-J.; Shao, Q.; Li, M.-H.; Zhou, Z.-L.; Xu, H.; Dai, H.X.; Yu, J.-Q. Organometallics 2018, 37, 2832.

(c) Yan, X.-B.; Gao, P.; Yang, H.-B.; Li, Y.-X.; Liu, X.-Y.; Liang, Y.-M. Tetrahedron 2014, 70, 8730.

[17] (a) Wang, T.; Xu, K.; Zhang, A.; Wang, W.; Liu, L. Chin. J. Org. Chem. 2018, 38, 259 (in Chinese).

(王涛, 许凯, 张安安, 王万里, 刘澜涛, 有机化学, 2018, 38, 259.)

(b) Wang, T.; Xu, K.; Liu, L.; Xie, H.; Li, Y.; Zhao, W-. X. Transition Met. Chem. 2016, 41, 525.

[18] Ouyang, K.; Xi, Z. Acta Chim. Sinica 2013, 71, 13 (in Chinese). (欧阳昆冰, 席振峰, 化学学报, 2013, 71, 13.)

[19] Shang, M.; Sun, S.-Z.; Dai, H.-X.; Yu, J.-Q. J. Am. Chem. Soc. 2014, 136, 3354. 